\title{
The Flux-Across-Surfaces Theorem for Short Range Potentials and Wave Functions without Energy Cutoffs
}

\author{
S. Teufel, D. Dürr, K. Münch-Berndl \\ Mathematisches Institut der Universität München, \\ Theresienstraße 39, 80333 München, Germany \\ e-mail: teufel@rz.mathematik.uni-muenchen.de
}

April 7, 1999

\begin{abstract}
.
The quantum probability flux of a particle integrated over time and a distant surface gives the probability for the particle crossing that surface at some time. The relation between these crossing probabilities and the usual formula for the scattering cross section is provided by the flux-acrosssurfaces theorem, which was conjectured by Combes. Newton and Shtokhamer. ${ }^{1}$ We prove the fluxacross-surfaces theorem for short range potentials and wave functions without energy cutoffs. The proof is based on the free flux-across-surfaces theorem (Daumer et. al.), ${ }^{2}$ and on smoothness properties of generalized eigenfunctions: It is shown that if the potential $V(x)$ decays like $|x|^{-\gamma}$ at infinity with $\gamma>n \in \mathbb{N}$ then the generalized eigenfunctions of the corresponding Hamiltonian $-\frac{1}{2} \Delta+V$ are $n-2$ times continuously differentiable with respect to the momentum variable.
\end{abstract}

PACS: $03.80,03.65 . \mathrm{N}, 02.30 . \mathrm{Nw}$

\section{Introduction}

Potential scattering theory is concerned with the long-time behavior of wave functions $\Psi_{t}$. Its relation to experiment, i.e. to the definition of the scattering cross section is however only rarely discussed. One such relation is provided by Dollard's scattering-into-cones theorem. ${ }^{3}$ It asserts that, assuming asymptotic completeness of the wave operators, the probability of finding a particle with a wave function $\Psi_{t}=$ $e^{-i H t} \Psi_{0} \in \mathcal{H}_{a c}(H)$, the absolutely continuous subspace for the Hamiltonian $H$, in the far future in a given cone $C \subset \mathbb{R}^{3}$ (with vertex at the origin) equals the probability that the quantum mechanical momentum of the asymptotic outgoing wave $W_{+}^{-1} \Psi_{0}$ lies in the same cone,

$$
\lim _{t \rightarrow \infty} \int_{C}\left|\Psi_{t}(x)\right|^{2} d x=\int_{C}\left|\widehat{W_{+}^{-1}} \Psi_{0}(k)\right|^{2} d k,
$$

where ${ }^{-}$denotes the Fourier transform, $W_{+}:=\mathrm{s}^{-} \lim _{t \rightarrow \infty} e^{i H t} e^{-i H_{0} t}$ is the wave operator and $H=H_{0}+V$ with the free Hamiltonian $H_{0}=-\frac{1}{2} \Delta$ (we choose units such that $\hbar=m=1$ ) and the potential $V$. The 
scattering-into-cones theorem is regarded as fundamental for quantum mechanical scattering theory. The expression for the differential cross-section $d \sigma / d \Omega=|f(\theta, \phi)|^{2}$ from the time-independent scattering theory can be derived from the right-hand side of (1). ${ }^{4}$

Combes, Newton and Shtokhamer ${ }^{1}$ observed, however, that what is relevant for scattering theory is a formula for the probability that the particle crosses some distant surface at some time during the scattering process. Heuristically, this probability should be given by integrating the quantum mechanical probability flux $j^{\Psi_{t}}:=\operatorname{Im}\left(\Psi_{t}^{*} \nabla \Psi_{t}\right)$ over the relevant time interval and this surface. ${ }^{2,5,6}$ Combes, Newton, and Shtokhamer hence conjectured the flux-across-surfaces theorem

$$
\lim _{R \rightarrow \infty} \int_{0}^{\infty} d t \int_{R \Sigma} j^{\Psi_{t}} \cdot n d \sigma=\int_{C_{\Sigma}}\left|\widehat{W_{+}^{-1}} \Psi_{0}(k)\right|^{2} d k
$$

where $\Sigma$ is a measurable subset of $S_{1}$, the sphere with radius $1, R \Sigma:=\left\{R x \in \mathbb{R}^{3}: x \in \Sigma\right\}$ and $C_{\Sigma}:=\left\{\lambda x \in \mathbb{R}^{3}: x \in \Sigma, \lambda \geq 0\right\}$ is the cone spanned by $\Sigma$. (2) was proven by Daumer, Dürr, Goldstein, and Zanghi ${ }^{2}$ for the case $\bar{V}=0$. Recently (2) has been established for a large class of short-range potentials by Amrein and Zuleta. ${ }^{7}$ For long-range potentials Amrein and Pearson ${ }^{8}$ showed that the left hand side of (2) equals the left hand side of (1). (In this case modified wave operators have to be introduced to define the right hand side of (1) and (2)). However, since the proofs in references 7 and 8 apply the usual time-dependent methods, they have to assume that $\widehat{W_{+}^{-1}} \Psi_{0}$ has compact support not containing the origin. Although this condition is a natural idealization of the experimental situation often encountered in scattering theory, and these wave functions form a dense set in $L^{2}$, there are no physical or mathematical reasons that (2) should hold only for this restricted class of wave functions. Furthermore there are situations, i.e. the decay of an unstable system, where the physically interesting wave functions do have momentum support at zero. But the set of wave functions for which (2) holds can not be enlarged by a simple limiting procedure in $L^{2}$, since the expression

$$
\int_{0}^{\infty} d t \int_{R \Sigma} j^{\Psi_{t}} \cdot n d \sigma
$$

is an unbounded sesquilinear form. Therefore the essential propagation estimates have to be proven directly for wave functions without energy cutoffs. Some results in this directions, so called $L^{p}$-estimates, have been established under rather restrictive conditions on the potential. ${ }^{9,10}$ However, these estimates alone are not sufficient to prove (2).

In this paper we will give an elementary proof of (2) for a class of wave functions without energy cutoffs. We must assume, however, that the potential is short-range with decay of order $|x|^{-4-\epsilon}, \epsilon>0$, at infinity and that it does not have a zero energy resonance or eigenvalue. Our proof as well as the proof in reference 7 are based on the results of the free case $(V=0)$ established in reference 2 . We employ stationary phase methods and the so called generalized eigenfunctions $\Phi(x, k)$, which are certain solutions of the stationary Schrödinger equation $\left(-\frac{1}{2} \Delta+V(x)\right) \Phi(x, k)=k^{2} \Phi(x, k), k \in \mathrm{IR}^{3}$, not belonging to $L^{2}\left(\mathbb{R}^{3}\right)$. This strategy of proof has been put forward in reference 5 . We need $\Phi(x, k)$ to be differentiable with respect to $k$ as well as to be uniformly bounded in both variables. Furthermore we need that $\sup _{k \in \mathbb{R} \backslash\{0\}}\left|\partial_{k_{l}} \Phi(x, k)\right| \leq c(1+|x|)$ for some constant $c$ and $l=1,2,3$.

In Section 2 the flux-across-surfaces theorem will be established under suitable conditions on the generalized eigenfunctions. In Section 3 we will prove a theorem on the regularity of the generalized eigenfunctions, which will, among other things, justify the assumptions made in Section $2: \Phi(x, k)$ is $n$ times partially differentiable with respect to $k$ if $V(x)=O\left(|x|^{-n-2-\epsilon}\right)$ for $|x| \rightarrow \infty$ and some $\epsilon>0$. 
Moreover, consider a family of Hamiltonians $H_{c}:=H_{0}+c V, c \in \mathbb{R}$. Then, if $V(x)=O\left(|x|^{-3-\epsilon}\right),{ }^{11}$ the eigenfunctions corresponding to $H_{c}$ are uniformly bounded and their partial derivatives of order $n$ with respect to $k$ grow not faster than $(1+|x|)^{n}$ except for a discrete set of constants $c \in \mathbb{R}$.

\section{The Flux-Across-Surfaces Theorem}

We start with notation. Points in position space will be denoted by $x \in \mathbb{R}^{3}$, points in momentum space by $k \in \mathbb{R}^{3}$. By $d x$ and $d k$ integration with respect to Lebesgue measure on $\mathbb{R}^{3}$ is understood. For $n \geq 2$, the following conditions on the potential $V$ will be denoted by $(\mathbf{V})_{n}$ :

$(\mathbf{V})_{n} \quad V: \mathbb{R}^{3} \rightarrow \mathbb{R}$ and

(i) $V$ is locally Hölder continuous except at a finite number of singularities. ${ }^{12}$

(ii) $V \in L^{2}\left(\mathbb{R}^{3}\right)$.

(iii) $|V(x)|=O\left(|x|^{-n-\epsilon}\right)$ for $|x| \rightarrow \infty$ and some $\epsilon>0$.

For $n=2$ these are the conditions of Ikebe. ${ }^{13}$ Under these conditions $H$ is self-adjoint on the domain of $H_{0}$. The absolutely continuous part of the spectrum is $[0, \infty)$. Furthermore $H$ has neither positive eigenvalues nor singular continuous spectrum. The wave operators $W_{ \pm}=s-\lim _{t \rightarrow \pm \infty} e^{-i H t} e^{i H_{0} t}$ exist and are complete, i.e. $\operatorname{Ran} W_{ \pm}=\mathcal{H}_{a c}(H)$.

The time dependent wave function will be denoted by $\Psi_{t}:=e^{-i H t} \Psi_{0}, \Psi_{0} \in L^{2}\left(\mathbb{R}^{3}\right)$. To simplify notation we will abbreviate $\Psi_{\text {out }}:=W_{+}^{-1} \Psi_{0}$ for the outgoing asymptotic wave. By $S$ we denote the set of Schwartz functions.

Zero is said to be a resonance of $H$ if there exists a solution $f$ of $-\frac{1}{2} \Delta f(x)+V(x) f(x)=0$ such that $(1+|x|)^{-\gamma} f(x) \in L^{2}\left(\mathbb{R}^{3}\right)$ for any $\gamma>\frac{1}{2}$ and not for $\gamma=0 .{ }^{14}$ The appearance of zero-energy resonances or eigenvalues is an exceptional event: $H_{c}=H_{0}+\mathrm{cV}$ can have a zero-energy resonance or eigenvalue only for $c$ in discrete subset of $\mathbb{I R}^{14}$

2.1 Theorem. Let the potential satisfy the condition $(\mathbf{V})_{4}$ and let zero be neither a resonance nor an eigenvalue of $H$. Let $\Psi_{\text {out }} \in S$. Then $\Psi_{t}=e^{-i H t} W_{+} \Psi_{\text {out }}$ is continuously differentiable except at the singularities of $V$ and for any measurable $\Sigma \subset S_{1}$ and any $T \in \mathbb{R}$

$$
\lim _{R \rightarrow \infty} \int_{T}^{\infty} d t \int_{R \Sigma} j^{\Psi_{t}}(x) \cdot n d \sigma=\lim _{R \rightarrow \infty} \int_{T}^{\infty} d t \int_{R \Sigma}\left|j^{\Psi_{t}}(x) \cdot n\right| d \sigma=\int_{C_{\Sigma}}\left|\widehat{\Psi}_{\text {out }}(k)\right|^{2} d k .
$$

2.2 Remark. The first equality in (3) shows that far away from the scattering center the flux is essentially outgoing, i.e.that there the particles cross spherical surfaces only once and do not return. Thus (3) yields the crossing probability of interest.

2.3 Remark. It would be of course more satisfactory if we could prove (3) under a suitably general condition on $\Psi_{0}$, not on $\Psi_{\text {out }}$. However, the set of wave functions $\Psi_{0}=W_{+} \Psi_{\text {out }}$ for which Theorem 2.1 holds is dense in $\mathcal{H}_{a c}(H)$ since $S$ is dense in $L^{2}$ and $W_{+}: L^{2} \rightarrow \mathcal{H}_{a c}(H)$ is unitary. For an explicit characterization of the domain $W_{+} S$ one would need suitable mapping properties of the wave operators. 
Some mapping properties for wave functions without energy cutoffs have been established by Yajima, ${ }^{10}$ however, they are not sufficient for our purpose.

2.4 Remark. Due to the so called intertwining property of the wave operators, $W_{ \pm} e^{-i H_{0} t}=e^{-i H t} W_{ \pm}$, and the fact that $S$ is left invariant under the free time evolution, the condition imposed on $\Psi$ in Theorem 2.1 is invariant under the full time evolution: $e^{-i H t} W_{+} S=W_{+} e^{-i H_{0} t} S=W_{+} S$.

As already mentioned we will make use of the generalized eigenfunctions $\Phi(x, k)$ which diagonalize $H$ in the same sense as the ordinary plane waves $\left\{e^{i k \cdot x}, k \in \mathbb{R}^{3}\right\}$ diagonalize $H_{0}$. We define $\Phi(x, k)$ and state the properties that we will use in the proof of Theorem 2.1 in a proposition:

2.5 Proposition. Let $V$ satisfy $(\mathbf{V})_{2}$. Then for any $k \in \mathbb{R}^{3} \backslash\{0\}$ there are unique continuous solutions $\Phi_{ \pm}(\cdot, k): \mathbb{R}^{3} \rightarrow \mathbb{C}$ of the Lippmann-Schwinger equations

$$
\Phi_{ \pm}(x, k)=e^{i k \cdot x}-\frac{1}{2 \pi} \int \frac{e^{\mp i|k||x-y|}}{|x-y|} V(y) \Phi_{ \pm}(y, k) d y
$$

with the boundary conditions $\lim _{|x| \rightarrow \infty}\left[\Phi_{ \pm}(x, k)-e^{i k \cdot x}\right]=0$, which are also classical solutions of the stationary Schrödinger equation

$$
\left[-\frac{1}{2} \Delta+V(x)\right] \Phi_{ \pm}(x, k)=\frac{k^{2}}{2} \Phi_{ \pm}(x, k),
$$

such that:

(i) For any compact $D \subset \mathbb{R}^{3} \backslash\{0\}$ the functions $\Phi_{ \pm}(\cdot, \cdot): \mathbb{R}^{3} \times D \rightarrow \mathbb{C}$ are uniformly continuous.

(ii) For any $f \in L^{2}\left(\mathbb{R}^{3}\right)$ the generalized Fourier transforms

$$
\left(\mathcal{F}_{ \pm} f\right)(k)=\frac{1}{(2 \pi)^{\frac{3}{2}}} \text { l.i.m. } \int \Phi_{ \pm}^{*}(x, k) f(x) d x
$$

exist in $L^{2}\left(\mathbb{R}^{3}\right) \cdot{ }^{15}$

(iii) $\operatorname{Ran} \mathcal{F}_{ \pm}=L^{2}\left(\mathbb{R}^{3}\right)$ and $\mathcal{F}_{ \pm}: \mathcal{H}_{a c}(H) \rightarrow L^{2}\left(\mathbb{R}^{3}\right)$ are unitary and the inverse of $\mathcal{F}_{ \pm}$is given by

$$
\left(\mathcal{F}_{ \pm}^{-1} f\right)(x)=\frac{1}{(2 \pi)^{\frac{3}{2}}} \text { l.i.m. } \int \Phi_{ \pm}(x, k) f(k) d k
$$

(iv) For any $f \in D(H) \cap \mathcal{H}_{a c}(H)$ we have

$$
H f(x)=\left(\mathcal{F}_{ \pm}^{-1} \frac{k^{2}}{2} \mathcal{F}_{ \pm} f\right)(x)=\frac{1}{(2 \pi)^{\frac{3}{2}}} \text { l.i.m. } \int \frac{k^{2}}{2} \Phi_{ \pm}(x, k)\left(\mathcal{F}_{ \pm} f\right)(k) d k
$$

and therefore for any $f \in \mathcal{H}_{a c}(H)$

$$
e^{-i H t} f(x)=\left(\mathcal{F}_{ \pm}^{-1} e^{-i \frac{k^{2}}{2} t} \mathcal{F}_{ \pm} f\right)(x)=\frac{1}{(2 \pi)^{\frac{3}{2}}} l . i . m . \int e^{-i \frac{k^{2} t}{2}} \Phi_{ \pm}(x, k)\left(\mathcal{F}_{ \pm} f\right)(k) d k
$$


(v) For any $f \in \mathcal{H}_{a c}(H)$ the relations $W_{ \pm} f=\mathcal{F}_{ \pm}^{-1} \mathcal{F} f$ hold, where $\mathcal{F}$ denotes the ordinary Fourier transform.

(vi) If $V$ satisfies $(\mathbf{V})_{3}$, then $\Phi_{ \pm}(x, k)$ are continuously differentiable with respect to $k$ for all $x \in \mathbb{R}^{3}$ except at $k=0$. The partial derivatives $\partial_{k_{l}} \Phi_{ \pm}(x, k)$ are continuous in $x$ and $k$.

If, in addition, zero is not an eigenvalue or resonance of $H$, then $\Phi_{ \pm}(x, k)$ is well defined and continuous for $x, k \in \mathbb{R}^{3}$,

$$
\sup _{x \in \mathbb{R}^{3}, k \in \mathbb{R}^{3}}\left|\Phi_{ \pm}(x, k)\right|<\infty
$$

and there is a $c<\infty$ such that

$$
\sup _{k \in \mathbb{R}^{3} \backslash\{0\}}\left|\frac{\partial}{\partial k_{l}} \Phi_{ \pm}(x, k)\right|<c(1+|x|)
$$

for $l=1,2,3$.

The proof of Proposition 2.5,(i)-(v) is due to Ikebe. ${ }^{13}$ (vi) is a special case of Theorem 3.1 on the regularity of generalized eigenfunctions which we shall state and prove in Section 3.

2.6 Remark. Similar eigenfunction expansions can be obtained also for potentials with slower decay, but then in general the continuity in $k$ will not hold any more. ${ }^{16}$

Proof [of Theorem 2.1]. Let $\Psi_{t}=e^{-i H t} W_{+} \Psi_{\text {out }}, \Psi_{\text {out }} \in S$. Using Proposition 2.5.(iv), (v) and $\eta(x, k):=\Phi_{+}(x, k)-e^{i k \cdot x}$ we have that

$$
\begin{aligned}
\Psi_{t}(x) & =\frac{1}{(2 \pi)^{\frac{3}{2}}} \int e^{-i \frac{k^{2} t}{2}} \widehat{\Psi}_{\text {out }}(k) \Phi_{+}(x, k) d k \\
& =\frac{1}{(2 \pi)^{\frac{3}{2}}} \int e^{-i \frac{k^{2} t}{2}} \widehat{\Psi}_{\text {out }}(k) e^{i k \cdot x} d k+\frac{1}{(2 \pi)^{\frac{3}{2}}} \int e^{-i \frac{k^{2} t}{2}} \widehat{\Psi}_{\text {out }}(k) \eta(x, k) d k \\
= & \alpha(x, t)+\beta(x, t) .
\end{aligned}
$$

The flux generated by this wave function is

$$
j^{\Psi_{t}}(x)=\operatorname{Im}\left(\alpha^{*} \nabla \alpha+\alpha^{*} \nabla \beta+\beta^{*} \nabla \alpha+\beta^{*} \nabla \beta\right),
$$

where the differentiability of $\alpha$ is obvious and that of $\beta$ will be established later.

The first part $j_{0}=\operatorname{Im}\left(\alpha^{*} \nabla \alpha\right)$ is the flux generated by the free time evolution of $\Psi_{\text {out }}$ and according to the free flux-across-surfaces theorem ${ }^{2}$

$$
\lim _{R \rightarrow \infty} \int_{T}^{\infty} d t \int_{R \Sigma} j_{0}(x, t) \cdot n d \sigma=\lim _{R \rightarrow \infty} \int_{T}^{\infty} d t \int_{R \Sigma}\left|j_{0}(x, t) \cdot n\right| d \sigma=\int_{C_{\Sigma}}\left|\widehat{\Psi}_{\text {out }}(k)\right|^{2} d k .
$$

Therefore to prove (3) we need only show that the last three terms in (9) do not contribute to the flux across distant surfaces, i.e. that for $j_{1}:=\operatorname{Im}\left(\alpha^{*} \nabla \beta+\beta^{*} \nabla \alpha+\beta^{*} \nabla \beta\right)$

$$
\lim _{R \rightarrow \infty} \int_{T}^{\infty} d t \int_{S_{R}}\left|j_{1}(x, t) \cdot n\right| d \sigma=0 .
$$


For some fixed $T>0$ this will follow from the estimates (which we shall prove below)

$$
\begin{array}{cc}
\sup _{x \in S_{R}}|\alpha(x, t)| \leq t^{-\frac{3}{2}} f_{1}(R, t) & \forall t \geq T \\
\sup _{x \in S_{R}}|\nabla \alpha(x, t)| \leq t^{-\frac{3}{2}} f_{2}(R, t) & \forall t \geq T,
\end{array}
$$

where there exists a $c<\infty$ such that $f_{i}(R, t)$ satisfy

$$
\lim _{R \rightarrow \infty} f_{i}(R, t)=0 \quad \forall t \geq T
$$

and

$$
\sup _{R \in[0, \infty), t \geq T} f_{i}(R, t)<c
$$

for $i=1,2$, and there is $R_{0} \geq 0$ such that

$$
\begin{array}{cl}
\sup _{x \in S_{R}}|\beta(x, t)| \leq c \frac{1}{R(t+R)}, & \forall R>0, \\
\sup _{x \in S_{R}}|\nabla \beta(x, t)| \leq c \frac{1}{R(t+R)}, & \forall R>R_{0}
\end{array}
$$

for $t>T$. Note that the constants in these estimates depend on $T$.

Using (11) and (16) we obtain by dominated convergence

$$
\begin{aligned}
\lim _{R \rightarrow \infty} \int_{T}^{\infty} \int_{S_{R}}\left|\operatorname{Im}\left(\alpha^{*} \nabla \beta\right) \cdot n\right| d \sigma d t & \leq \lim _{R \rightarrow \infty} 4 \pi \int_{T}^{\infty} \sup _{x \in S_{R}} R^{2}|\alpha||\nabla \beta| d t \\
& \leq c \lim _{R \rightarrow \infty} \int_{T}^{\infty} \frac{R^{2} f_{1}(R, t)}{t^{\frac{3}{2}} R(t+R)} d t \\
& =c \int_{T}^{\infty} \lim _{R \rightarrow \infty} \frac{R f_{1}(R, t)}{t^{\frac{3}{2}}(t+R)} d t=0
\end{aligned}
$$

for $T>0$ where we observed that the integrand in (17) is bounded by an integrable function uniformly in $R$,

$$
\frac{R f_{1}(R, t)}{t^{\frac{3}{2}}(t+R)} \leq c t^{-\frac{3}{2}}
$$

The terms $\left|\beta^{*} \nabla \alpha\right|$ and $\left|\beta^{*} \nabla \beta\right|$ can be treated analogously and thus (10) holds for positive times $T$.

According to Remark 2.4, the set of wave functions for which (3) holds as well as the right hand side of (3) are invariant under finite time shifts: 


$$
\begin{aligned}
\lim _{R \rightarrow \infty} \int_{T}^{\infty} d t \int_{R \Sigma} j^{\Psi t}(x) \cdot n d \sigma & =\lim _{R \rightarrow \infty} \int_{\widetilde{T}}^{\infty} d t \int_{R \Sigma} j^{\Psi_{t+T}-\widetilde{T}}(x) \cdot n d \sigma \\
& =\int_{C_{\Sigma}}\left|e^{-i \frac{k^{2}}{2}(T-\widetilde{T})} \hat{\Psi}_{\text {out }}(k)\right|^{2} d k=\int_{C_{\Sigma}}\left|\widehat{\Psi}_{\text {out }}(k)\right|^{2} d k
\end{aligned}
$$

Therefore if (3) holds (for all $\Psi_{\text {out }} \in S$ ) for some fixed $T$, then (3) will hold for all $T$; hence (3) is proved for all $T$.

We turn now to the proof of the estimates (11-16). Recalling that $\alpha(x, t)=\left(e^{-i H_{0} t} \Psi_{\text {out }}\right)(x)$ and, since $\nabla$ commutes with the free time evolution, $\nabla \alpha(x, t)=\left(e^{-i H_{0} t} \nabla \Psi_{\text {out }}\right)(x)$, we can write

$$
\alpha(x, t)=\frac{1}{(2 \pi i t)^{\frac{3}{2}}} \int e^{i \frac{|x-y|^{2}}{2 t}} \Psi_{\text {out }}(y) d y
$$

and

$$
\nabla \alpha(x, t)=\frac{1}{(2 \pi i t)^{\frac{3}{2}}} \int e^{i \frac{|x-y|^{2}}{2 t}} \nabla \Psi_{\text {out }}(y) d y .
$$

(11-14) are now immediate consequences of (18) and (19) and the fact that, for every fixed $t \geq T, \alpha(x, t)$ and $\nabla \alpha(x, t)$ are Schwartz functions.

By (4) $\eta(x, k)=\Phi_{+}(x, k)-e^{i k \cdot x}=-\frac{1}{2 \pi} \int \frac{e^{-i|k||x-y|}}{|x-y|} V(y) \Phi_{+}(y, k) d y$ and therefore

$$
\begin{aligned}
\beta(x, t) & =\frac{1}{(2 \pi)^{\frac{3}{2}}} \int e^{-i \frac{k^{2} t}{2}} \widehat{\Psi}_{\text {out }}(k) \eta(x, k) d k \\
& =-\frac{1}{(2 \pi)^{\frac{5}{2}}} \int e^{-i \frac{k^{2} t}{2}} \widehat{\Psi}_{\text {out }}(k)\left[\int \frac{e^{-i|k||x-y|}}{|x-y|} V(y) \Phi_{+}(y, k) d y\right] d k \\
& =-\frac{1}{(2 \pi)^{\frac{5}{2}}} \int \frac{V(y)}{|x-y|} \int e^{-i\left(\frac{k^{2} t}{2}+|k||x-y|\right)} \widehat{\Psi}_{\text {out }}(k) \Phi_{+}(y, k) d k d y \\
= & -\frac{1}{(2 \pi)^{\frac{5}{2}}} \int \frac{V(y)}{|x-y|} f(x, y, t) d y
\end{aligned}
$$

where

$$
f(x, y, t):=\int e^{-i\left(\frac{k^{2} t}{2}+|k||x-y|\right)} \hat{\Psi}_{\text {out }}(k) \Phi_{+}(y, k) d k .
$$

The change of order of integration in (20) is justified by Fubini's theorem. We shall now apply "stationary phase" methods to estimate (21). We set

$$
\begin{aligned}
\chi & :=\frac{\frac{k^{2} t}{2}+|k||x-y|}{\frac{t}{2}+|x-y|} \\
\chi^{\prime} & =\frac{d}{d|k|} \chi=\frac{|k| t+|x-y|}{\frac{t}{2}+|x-y|}=\frac{|k|+|x-y| t^{-1}}{\frac{1}{2}+|x-y| t^{-1}} \geq \min (1,2|k|) \\
\omega & :=\frac{t}{2}+|x-y| .
\end{aligned}
$$


In the following' will denote differentiation with respect to $|k|$. Introducing spherical coordinates, with $d \Omega$ denoting Lebesgue measure on the unit sphere, we estimate (21):

$$
\begin{aligned}
|f(x, y, t)| & =\left.\left|\int \frac{1}{\omega \chi^{\prime}}\left[\frac{d}{d|k|} e^{-i \omega \chi}\right] \widehat{\Psi}_{\text {out }}(k) \Phi_{+}(y, k)\right| k\right|^{2} d|k| d \Omega(k) \mid \\
& =\left|\frac{1}{\omega} \int e^{-i \omega \chi} \frac{d}{d|k|}\left[\frac{1}{\chi^{\prime}} \widehat{\Psi}_{\text {out }}(k) \Phi_{+}(y, k)|k|^{2}\right] d\right| k|d \Omega(k)| \\
& \leq \frac{1}{\omega} \int\left|\frac{d}{d|k|}\left[\frac{1}{\chi^{\prime}} \widehat{\Psi}_{\text {out }}(k) \Phi_{+}(y, k)|k|^{2}\right]\right| d|k| d \Omega(k) .
\end{aligned}
$$

For the second equality in (22) the boundary term from the partial integration at $|k|=\infty$ vanishes since $\chi^{\prime}-1 \leq \max \left(1, \frac{1}{2|k|}\right), \lim _{|k| \rightarrow \infty}|k|^{2} \widehat{\Psi}_{\text {out }}(k)=0$, and $\Phi_{+}$is bounded according to Proposition 2.5.(vi). The boundary term at $|k|=0$ vanishes since $\hat{\Psi}_{\text {out }}$ and $\Phi_{+}$are bounded and $\chi^{\prime}-1|k|^{2} \leq \max \left(|k|^{2}, \frac{|k|}{2}\right)$. Note that the differentiability of $\Phi_{+}$is ensured by Proposition 2.5.(vi). Next, observe that

$$
\begin{aligned}
\left|\frac{d}{d|k|}\left[\frac{1}{\chi^{\prime}} \widehat{\Psi}_{\text {out }} \Phi_{+}|k|^{2}\right]\right| \leq & \left.\left|\frac{1}{\chi^{\prime 2}} \chi^{\prime \prime} \widehat{\Psi}_{\text {out }} \Phi_{+}\right| k\right|^{2}|+| \frac{1}{\chi^{\prime}} \widehat{\Psi}_{\text {out }}^{\prime} \Phi_{+}|k|^{2} \mid \\
& +\left.\left|\frac{1}{\chi^{\prime}} \widehat{\Psi}_{\text {out }} \Phi_{+}^{\prime}\right| k\right|^{2}|+| \frac{1}{\chi^{\prime}} \widehat{\Psi}_{\text {out }} \Phi_{+} 2|k| \mid .
\end{aligned}
$$

Since $\chi^{\prime \prime}=\left(\frac{1}{2}+|x-y| t^{-1}\right)^{-1} \leq 2$, we obtain for the first term

$$
\begin{aligned}
\int\left|\frac{1}{\chi^{\prime 2}} \chi^{\prime \prime} \widehat{\Psi}_{\text {out }} \Phi_{+}\right| d k & \leq \\
\sup _{y, k \in \mathbb{R}^{3}}\left|\Phi_{+}(y, k)\right| & \left(\int_{k \mid<\frac{1}{2}} \frac{\left|\hat{\Psi}_{\text {out }}(k)\right|}{2|k|^{2}} d k+\int_{|k| \geq \frac{1}{2}} 2\left|\widehat{\Psi}_{\text {out }}(k)\right| d k\right) \leq c_{1} .
\end{aligned}
$$

Analogously we get for the second and fourth term in (23)

$$
\int\left|\frac{1}{\chi^{\prime}} \widehat{\Psi}_{\text {out }}^{\prime} \Phi_{+}\right| d k \leq c_{2} \quad \text { and } \quad \int\left|\frac{2}{\chi^{\prime}|k|} \widehat{\Psi}_{\text {out }} \Phi_{+}\right| d k \leq c_{4}
$$

By Proposition 2.5.(vi) the third term satisfies a bound linear in $|y|$ :

$$
\int\left|\frac{1}{\chi^{\prime}} \widehat{\Psi}_{\text {out }} \Phi_{+}^{\prime}\right| d k \leq \widetilde{c}_{3} \sup _{k \in \mathbb{R}^{3} \backslash\{0\}}\left|\Phi_{+}^{\prime}(y, k)\right| \leq c_{3}(1+|y|) .
$$

Combining the four estimates we arrive at

$$
|f(x, y, t)| \leq c \frac{1}{\omega}(1+|y|)=c \frac{1+|y|}{\frac{t}{2}+|x-y|},
$$

which inserted into (20) yields 


$$
\begin{aligned}
\sup _{x \in S_{R}}|\beta(x, t)| & =\sup _{x \in S_{R}}\left|\frac{1}{(2 \pi)^{\frac{5}{2}}} \int \frac{V(y)}{|x-y|} f(x, y, t) d y\right| \\
& \leq c \sup _{x \in S_{R}} \int \frac{|V(y)|(1+|y|)}{|x-y|\left(\frac{t}{2}+|x-y|\right)} d y .
\end{aligned}
$$

Now, substituting $z=x-y$,

$$
\begin{aligned}
\int \frac{|V(y)|(1+|y|)}{|x-y|\left(\frac{t}{2}+|x-y|\right)} d y= & \int_{|z|<\frac{|x|}{2}} \frac{|V(x-z)|(1+|x-z|)}{|z|\left(\frac{t}{2}+|z|\right)} d z \\
& +\int_{|x-y| \geq \frac{|x|}{2}} \frac{|V(y)|(1+|y|)}{|x-y|\left(\frac{t}{2}+|x-y|\right)} d y \\
\leq & \sup _{z \in B}|V(x-z)|(1+|x-z|) \int_{0}^{\frac{|x|}{2}} \frac{4 \pi|z|^{2}}{|z|\left(\frac{t}{2}+|z|\right)} d|z| \\
& +\frac{1}{\frac{|x|}{2}\left(\frac{t}{2}+\frac{|x|}{2}\right)} \int|V(y)|(1+|y|) d y,
\end{aligned}
$$

where $B_{r}$ denotes the ball with radius $r$ in $\mathbb{R}^{3}$ centered at the origin. Since $V(x)=O\left(|x|^{-4-\epsilon}\right)$ for some $\epsilon>0$,

$$
\sup _{z \in B}|V(x-z)|(1+|x-z|) \leq c|x|^{-3}
$$

for $|x|$ sufficiently large. Using

$$
\int_{0}^{\delta} \frac{z}{t+z} d z=\delta+t \ln \left(\frac{t}{t+\delta}\right) \leq \delta+t\left(\frac{t}{t+\delta}-1\right)=\frac{\delta^{2}}{t+\delta}
$$

we compute

$$
\int_{0}^{\frac{|x|}{2}} \frac{|z|^{2}}{|z|\left(\frac{t}{2}+|z|\right)} d|z| \leq \frac{1}{2} \frac{|x|^{2}}{t+|x|} .
$$

Finally $\int|V(y)|(1+|y|) d y<\infty$ so that altogether

$$
\begin{aligned}
\sup _{x \in S_{R}}|\beta(x, t)| & \leq c \sup _{x \in S_{R}}\left(|x|^{-3} \frac{|x|^{2}}{t+|x|}+\frac{1}{|x|(t+|x|)}\right) \\
& =\frac{c}{R(t+R)} .
\end{aligned}
$$


We now show that the same bound holds for $\sup _{x \in S_{R}}|\nabla \beta(x, t)|$ and $R>R_{0}$, where $R_{0}$ is chosen such that all singularities of $V$ lie in the ball with radius $R_{0}$. Then

$$
\begin{aligned}
|\nabla \beta(x, t)| \leq & \frac{1}{(2 \pi)^{\frac{5}{2}}}\left|\int \frac{V(y)}{|x-y|^{2}} \int e^{-i\left(\frac{k^{2} t}{2}+|k||x-y|\right)} \hat{\Psi}_{\text {out }}(k) \Phi_{+}(y, k) d k d y\right| \\
& +\frac{1}{(2 \pi)^{\frac{5}{2}}}\left|\int \frac{V(y)}{|x-y|} \int e^{-i\left(\frac{k^{2} t}{2}+|k||x-y|\right)}\right| k\left|\widehat{\Psi}_{\text {out }}(k) \Phi_{+}(y, k) d k d y\right|
\end{aligned}
$$

where the exchange of differentiation and integration will be justified below. The second term can be treated analogously to $|\beta(x, t)|$, since also $|k| \widehat{\Psi}_{\text {out }}(k) \in S$. The first term can as well be estimated along the same lines: in Equation (26) $|x-y|^{2}$ will appear in the denominator instead of $|x-y|$, which leads to a stronger bound than (27).

To get (28) from (20) we note that according to Proposition $2.5 \Phi(\cdot, k)$ is a classical solution of the stationary Schrödinger equation. Thus $\Phi(\cdot, k)$ as well as $\eta(\cdot, k)$ are differentiable with respect to $x$ except at the singularities of $V$. We will show that

$$
\begin{aligned}
\nabla_{x} \eta(x, k) & =\nabla_{x}\left(-\frac{1}{2 \pi} \int \frac{e^{-i|k||x-y|}}{|x-y|} V(y) \Phi_{+}(y, k) d y\right) \\
& =-\frac{1}{2 \pi} \int \nabla_{x}\left(\frac{e^{-i|k||x-y|}}{|x-y|}\right) V(y) \Phi_{+}(y, k) d y
\end{aligned}
$$

and that therefore

$$
\left|\nabla_{x} \eta(x, k)\right| \leq c_{1}+c_{2}|k|
$$

for some $c_{1}, c_{2}<\infty$. Then changing the order of differentiation and integration in the first line of (20) is justified by dominated convergence and (28) follows for all $x$ which are not singularities of $V$.

To get (29) for some $x_{0} \in \mathbb{R}^{3}$ which is no singularity of $V$, we split the domain of integration into $B_{2 R}\left(x_{0}\right):=\left\{y \in \mathbb{R}^{3}:\left|x_{0}-y\right| \leq 2 R\right\}$ and its complement $B_{2 R}^{c}\left(x_{0}\right)$, where $R$ is chosen such that $B_{2 R}\left(x_{0}\right)$ contains no singularity of $V$. Then one can change the order of integration and differentiation in the $B_{2 R}^{c}\left(x_{0}\right)$ term, since there the integrand is bounded by an integrable function uniformly in $x$ for $x \in B_{R}\left(x_{0}\right)$. To see that the $B_{2 R}\left(x_{0}\right)$ term can be made arbitrary small by appropriately choosing $R$, we write down the difference quotient for this term. Using that $\sup _{y \in B_{2 R}\left(x_{0}\right)}\left(V(y) \Phi_{+}(y, k)\right) \leq c_{k}<\infty$ and that $\left|r e^{i \theta}-(r+\Delta r) e^{i(\theta+\Delta \theta)}\right| \leq|r \Delta \theta|+|\Delta r|$ we compute

$$
\begin{array}{r}
\lim _{|\epsilon| \rightarrow 0} \frac{1}{|\epsilon|} \int_{B_{2 \Omega}\left(x_{0}\right)}\left(\frac{e^{-i|k||x+\epsilon-y|}}{|x+\epsilon-y|}-\frac{e^{-i|k||x-y|}}{|x-y|}\right) V(y) \Phi_{+}(y, k) d y \mid \\
\leq \lim _{|\epsilon| \rightarrow 0} \frac{1}{|\epsilon|} c_{k} \int_{B_{2 R}\left(x_{0}\right)}\left|\frac{e^{-i|k||x+\epsilon-y|}|x-y|-e^{-i|k||x-y|}|x+\epsilon-y|}{|x+\epsilon-y||x-y|}\right| d y \\
\leq \lim _{|\epsilon| \rightarrow 0} \frac{1}{|\epsilon|} c_{k} \int_{B_{2 R}\left(x_{0}\right)} \frac{|x-y||k||\epsilon|+|\epsilon|}{|x+\epsilon-y||x-y|} d y
\end{array}
$$




$$
\leq \lim _{|\epsilon| \rightarrow 0} c_{k} \int_{B_{2 R}\left(x_{0}\right)}\left(\frac{|k|}{|x+\epsilon-y|}+\frac{1}{|x+\epsilon-y||x-y|}\right) d y \leq c_{k} 12 \pi R,
$$

where the last inequality is justified by elementary integrations. The bound (30) can be obtained by a simple calculation.

From that we also conclude that $\Psi_{t}(x)$ is differentiable outside the singularities of $V(x)$ since $\alpha$ and $\beta$ are.

2.7 Remark. We used the domain $\Psi_{\text {out }} \in S$ to simplify the proof and avoid tedious estimates. A more detailed analysis of the proof shows that Theorem 2.1 also holds for $\Psi_{\text {out }} \in L^{2}$ such that $\left(1+k^{2}\right)^{\frac{q+p}{2}}(1+\Delta)^{\frac{p}{2}} \widehat{\Psi}_{\text {out }} \in L^{2}$ for some $p>\frac{7}{2}$ and $q>\frac{9}{2}$. Then $\left(1+k^{2}\right)^{\frac{q}{2}}(1+\Delta)^{\frac{p}{2}} e^{-i \frac{k^{2}}{2} t} \widehat{\Psi}_{\text {out }} \in L^{2}$ for all $t \in \mathbb{R}$ and this is enough regularity to prove the free theorem as well as our estimates.

\section{Regularity of the Generalized Eigenfunctions}

In this Section we will prove a theorem about the generalized eigenfunctions that connects the differentiability of $\Phi(x, k)$ with respect to $k$ with the behavior of the potential at infinity and gives uniform bounds on $\Phi(x, k)$ and its partial derivatives. At the end of the section we state two simple corollaries that show other applications of our results.

3.1 Theorem. Let the potential satisfy the condition $(\mathbf{V})_{\mathbf{n}}$ for some $n \geq 3, n \in \mathbb{N}$. Then

(i) $\Phi_{ \pm}(x, \cdot) \in C^{n-2}\left(\mathbb{R}^{3} \backslash\{0\}\right)$ for all $x \in \mathbb{R}^{3}$ and the partial derivatives $\partial_{k}^{\alpha} \Phi_{ \pm}(x, k),|\alpha| \leq n-2$, are continuous with respect to $x$ and $k^{17}$

(ii) If, in addition, zero is not an eigenvalue or a resonance of $H$, then

$$
\sup _{x \in \mathbb{R}^{3}, k \in \mathbb{R}^{3}}\left|\Phi_{ \pm}(x, k)\right|<\infty
$$

and for any $\alpha$ with $|\alpha| \leq n-2$ there is a $c_{\alpha}<\infty$ such that

$$
\sup _{k \in \mathbb{R}^{3} \backslash\{0\}}\left|\partial_{k}^{\alpha} \Phi_{ \pm}(x, k)\right|<c_{\alpha}(1+|x|)^{|\alpha|} .
$$

3.2 Remark. Proposition 2.5.(vi) follows from Theorem 3.1 by taking $n=3$.

Proof [of Theorem 3.1]. To simplify notation we will give the proof for $\Phi_{+}(x, k)=: \Phi(x, k)$ since the proof for $\Phi_{-}(x, k)$ is exactly the same apart from the change of some signs.

The structure of the proof will be as follows: First we introduce some notation and results from Ikebe and Povzner that we will use frequently. Then part (i) of Theorem 3.1 is shown for $|\alpha|=1$ involving several lemmas and results proven in the appendix. The generalization to $|\alpha| \geq 1$ will be sketched afterwards. 
In the proof of part (ii) of Theorem 3.1 we will establish boundedness of $\Phi(x, k)$ for $k$ near zero and for $|k| \rightarrow \infty$ separately in two propositions.

We start with an investigation of Equation (4). If $\Phi(x, k)=e^{i k \cdot x}+\eta(x, k)$ is a continuous solution of the Lippmann-Schwinger equation (4) with $\lim _{|x| \rightarrow \infty} \eta(x, k)=0$ for $k \in \mathbb{R}^{3}$, then $\eta(x, k)$ is a solution of the integral equation

$$
\eta(x, k)=-\frac{1}{2 \pi} \int \frac{e^{-i|k||x-y|}}{|x-y|} V(y)\left[e^{i k \cdot y}+\eta(y, k)\right] d y
$$

and $\eta(\cdot, k) \in C_{\infty}\left(\mathbb{R}^{3}\right)$. Therefore Equation $(31)$ is examined on the Banach space $B=C_{\infty}\left(\mathbb{R}^{3}\right)$, the set of continuous functions vanishing at infinity, equipped with the norm $\|f\|_{B}=\sup _{x \in \mathbb{R}^{3}}|f(x)| . \mathcal{L}(B)$ denotes the space of bounded linear operators mapping $B$ into itself, equipped with the operator norm. Following Ikebe ${ }^{13}$ we define the linear operators $T_{k} \in \mathcal{L}(B), k \in \mathbb{R}^{3}$, by

$$
\left(T_{k} f\right)(x)=-\frac{1}{2 \pi} \int \frac{e^{-i|k||x-y|}}{|x-y|} V(y) f(y) d y .
$$

Since we will make use of some results of Ikebe and Povzner, we state them as a lemma:

3.3 Lemma. Let the potential satisfy the condition $(\mathbf{V})_{\mathbf{3}}$. Then:

(i) The operator $T_{k} \in \mathcal{L}(B)$ defined in (32) is compact for all $k \in \mathbb{R}^{3}$.

(ii) Let $f(x)$ be a bounded continuous function on $\mathbb{R}^{3}$, then

$$
h(x):=-\frac{1}{2 \pi} \int \frac{e^{-i|k||x-y|}}{|x-y|} V(y) f(y) d y
$$

is an element of $B$ for all $k \in \mathbb{R}^{3}$ and $h(x)=O\left(|x|^{-1}\right)$ for $|x| \rightarrow \infty$.

(iii) Let

$$
g(x, k):=-\frac{1}{2 \pi} \int \frac{e^{-i|k||x-y|}}{|x-y|} V(y) e^{i k \cdot y} d y=\left(T_{k} e^{i k \cdots}\right)(x),
$$

then $g(\cdot, k) \in B$ for all $k \in \mathbb{R}^{3}$ and $g(\cdot, k)$ is continuous with respect to $k$.

(iv) Let $f(\cdot, k) \in B$ be a solution of the homogeneous equation $f(\cdot, k)=T_{k} f(\cdot, k)$ for $k \in \mathbb{R}^{3}$. If $|k|>0$ then $f=0$ and if $k=0$ then $\left(-\frac{1}{2} \Delta+V(x)\right) f(x, 0)=0$.

(v) The map $T: \mathbb{R}^{3} \rightarrow \mathcal{L}(B), k \mapsto T_{k}$ is continuous.

For the proofs of (i), (ii), (iii) and (iv) see Ikebe ${ }^{13}$, for the proof of (v) see Povzner ${ }^{18}$.

Since we will use similar reasoning, we will briefly repeat Ikebe's proof of the existence of continuous solutions of Equation (31) starting from Lemma 3.3. Equation (31) now reads

$$
\eta(\cdot, k)=g(\cdot, k)+T_{k} \eta(\cdot, k) .
$$

According to Lemma 3.3.(iv) the homogeneous equation $\eta(\cdot, k)=T_{k} \eta(\cdot, k)$ has only the trivial solution $\eta(x, k)=0$ if $k \neq 0$. Thus 1 is not an eigenvalue of $T_{k}$ and therefore 1 is in the resolvent set since $T_{k}$ is compact, ${ }^{19}$ i.e. $\left(1-T_{k}\right)^{-1} \in \mathcal{L}(B)$ exists. The unique solution of $(31)$ for $|k|>0$ is then given by 


$$
\eta(\cdot, k)=\left(1-T_{k}\right)^{-1} g(\cdot, k) .
$$

Since $\mathcal{L}(B)$ is a Banach algebra in which the map $A \mapsto A^{-1}$ is continuous, ${ }^{19}$ from Lemma 3.3.(v) it follows that $\left(1-T_{k}\right)^{-1}$ is continuous in $k$. Thus, since according to Lemma 3.3.(iii) $g(\cdot, k)$ is continuous with respect to $k$, we have that $\eta(x, k)$ is continuous with respect to $k$.

We will now prove part (i) of Theorem 3.1 for $|\alpha|=1$ and assume $(\mathbf{V})_{\mathbf{3}}$. The generalization to $|\alpha|>1$ will then be immediate. Consider arbitrary $l \in\{1,2,3\}$ and $k^{0} \in \mathbb{R}^{3} \backslash\{0\}$. We use the following notation: $k_{l}$ denotes the $l$-th cartesian coordinate of a vector $k \in \mathbb{R}^{3}$ and $k_{\tau}$ the tuple of the other coordinates. Symbolically we will write $k=\left(k_{l}, k_{\bar{l}}\right)$.

By (formally) differentiating (31) we obtain

$$
\begin{aligned}
\frac{\partial}{\partial k_{l}} \eta(x, k)= & \frac{\partial}{\partial k_{l}} g(x, k)+\frac{i}{2 \pi} \frac{k_{l}}{|k|} \int e^{-i|k||x-y|} V(y) \eta(y, k) d y \\
& -\frac{1}{2 \pi} \int \frac{e^{-i|k||x-y|}}{|x-y|} V(y) \frac{\partial}{\partial k_{l}} \eta(y, k) d y .
\end{aligned}
$$

Assume that for $k_{l} \in I_{l}:=\left[k_{l}^{0}-\delta_{l}, k_{l}^{0}+\delta_{l}\right]$ and $k_{\bar{l}} \in I_{\bar{l}}:=\left[k_{\bar{l}}^{0}-\delta_{\bar{l}}, k_{\bar{l}}^{0}+\delta_{\bar{l}}\right]$ where $\delta_{l}$ and $\delta_{\bar{l}}$ are chosen such that in particular $0 \notin I:=I_{l} \times I_{\bar{l}}$, the equation

$$
\begin{aligned}
\xi(x, k)= & \frac{\partial}{\partial k_{l}} g(x, k)+\frac{i}{2 \pi} \frac{k_{l}}{|k|} \int e^{-i|k||x-y|} V(y)\left[\int_{k_{l}^{0}}^{k_{l}} \xi\left(y,\left(k_{l}^{\prime}, k_{\bar{l}}\right)\right) d k_{l}^{\prime}+\eta\left(y,\left(k_{l}^{0}, k_{\bar{l}}\right)\right)\right] d y \\
& -\frac{1}{2 \pi} \int \frac{e^{-i|k||x-y|}}{|x-y|} V(y) \xi(y, k) d y,
\end{aligned}
$$

which arises from (35) by substituting $\eta(x, k)=\int_{k_{l}^{0}}^{k_{l}} \xi\left(x,\left(k_{l}^{\prime}, k_{\bar{l}}\right)\right) d k_{l}^{\prime}+\eta\left(x,\left(k_{l}^{0}, k_{\bar{l}}\right)\right)$, has a continuous solution $\xi(x, k)$. Integrating (36) with respect to $k_{l}$ and using Fubini's theorem we get

$$
\begin{aligned}
\int_{k_{l}^{0}}^{k_{l}} \xi\left(x,\left(k_{l}^{\prime}, k_{\bar{l}}\right)\right) d k_{l}^{\prime}= & g(x, k)-g\left(x,\left(k_{l}^{0}, k_{\bar{l}}\right)\right) \\
& -\frac{1}{2 \pi} \int V(y)\left[\frac{e^{-i|k||x-y|}}{|x-y|}\left(\int_{k_{l}^{0}}^{k_{l}^{\prime}} \xi\left(y,\left(k_{l}^{\prime \prime}, k_{\bar{l}}\right)\right) d k_{l}^{\prime \prime}+\eta\left(y,\left(k_{l}^{0}, k_{\bar{l}}\right)\right)\right)\right]_{k_{l}^{0}}^{k_{l}} d y \\
= & g(x, k)-g\left(x,\left(k_{l}^{0}, k_{\bar{l}}\right)\right) \\
& +\frac{1}{2 \pi} \int \frac{e^{-i\left|\left(k_{l}^{0}, k_{\bar{l}}\right)\right| x-y \mid}}{|x-y|} V(y) \eta\left(y,\left(k_{l}^{0}, k_{\bar{l}}\right)\right) d y \\
& -\frac{1}{2 \pi} \int \frac{e^{-i|k||x-y|}}{|x-y|} V(y)\left(\int_{k_{l}^{0}}^{k_{l}} \xi\left(y,\left(k_{l}^{\prime}, k_{\bar{l}}\right)\right) d k_{l}^{\prime}+\eta\left(y,\left(k_{l}^{0}, k_{\bar{l}}\right)\right)\right) d y .
\end{aligned}
$$


Since $\eta\left(x,\left(k_{l}^{0}, k_{\bar{l}}\right)\right)$ is a solution of (31) the second and third term of the right hand side of (37) combine to $-\eta\left(x,\left(k_{l}^{0}, k_{l}\right)\right)$. Therefore $(37)$ simply reads

$$
\begin{aligned}
& \eta\left(x,\left(k_{l}^{0}, k_{\bar{l}}\right)\right)+\int_{k_{l}^{0}}^{k_{l}} \xi\left(x,\left(k_{l}^{\prime}, k_{\bar{l}}\right)\right) d k_{l}^{\prime}= \\
& \quad g(x, k)-\frac{1}{2 \pi} \int \frac{e^{-i|k||x-y|}}{|x-y|} V(y)\left(\eta\left(y,\left(k_{l}^{0}, k_{\bar{l}}\right)\right)+\int_{k_{l}^{0}}^{k_{l}} \xi\left(y,\left(k_{l}^{\prime}, k_{\bar{l}}\right)\right) d k_{l}^{\prime}\right) d y .
\end{aligned}
$$

In other words, if $\xi(x, k)$ is a continuous solution of $(36)$ then the function $f(x, k)=\eta\left(x,\left(k_{l}^{0}, k_{l}\right)\right)+$ $\int_{k_{l}^{0}}^{k_{l}} \xi\left(x,\left(k_{l}^{\prime}, k_{\bar{l}}\right)\right) d k_{l}^{\prime}$ is a solution of Equation (31). Since (31) has a unique solution in $B$ we may conclude that $f(x, k)=\eta(x, k)$, i.e. that $\partial_{k_{l}} \eta(x, k)=\xi(x, k)$ for $x \in \mathbb{R}^{3}$ and $k \in I$ once we have shown that $f(\cdot, k) \in B$.

We show now that equation (36) has a solution $\xi(x, k)$ which is continuous with respect to $x \in \mathbb{R}^{3}$ and $k \in I$ such that $\eta\left(\cdot,\left(k_{l}^{0}, k_{\bar{l}}\right)\right)+\int_{k_{l}^{0}}^{k_{l}} \xi\left(\cdot,\left(k_{l}^{\prime}, k_{\bar{l}}\right)\right) d k_{l}^{\prime} \in B$. From the physical argument that $\Phi(x, k) \sim$ $e^{i k \cdot x}+\frac{e^{i|k||x|}}{|x|}$ for $|x| \rightarrow \infty$ we expect $\left|\nabla_{k} \eta(x, k)\right| \sim e^{i|k||x|},|x| \rightarrow \infty$, to be a uniformly bounded function, but we will only show that $\left|\nabla_{k} \eta(x, k)\right| \leq c(1+|x|)^{s}$ for any $s>0$. We start by multiplying equation (36) by $\langle x\rangle^{-s}:=(1+|x|)^{-s}, s>0$ :

$$
\begin{aligned}
\tilde{\xi}(x, k)= & \langle x\rangle^{-s} \partial_{k_{l}} g(x, k)-\frac{1}{2 \pi} \int \frac{e^{-i|k||x-y|}}{\langle x\rangle^{s}|x-y|}\langle y\rangle^{s} V(y) \tilde{\xi}(y, k) d y \\
& +\frac{i}{2 \pi} \frac{k_{l}}{|k|} \int \frac{e^{-i|k||x-y|}}{\langle x\rangle^{s}}\langle y\rangle^{s} V(y)\left[\int_{k_{l}^{0}}^{k_{l}} \tilde{\xi}\left(y,\left(k_{l}^{\prime}, k_{\bar{l}}\right)\right) d k_{l}^{\prime}+\langle y\rangle^{-s} \eta\left(y,\left(k_{l}^{0}, k_{\bar{l}}\right)\right)\right] d y .
\end{aligned}
$$

To see that $\xi(x, k)=\langle x\rangle^{s} \tilde{\xi}(x, k) \leq c(1+|x|)^{s}$, we show that $(38)$ has a unique solution in

$$
\widetilde{B}:=\left\{f(x, k) \in C\left(\mathbb{R}^{3} \times I\right): \lim _{|x| \rightarrow \infty} \sup _{k \in I}|f(x, k)|=0\right\}
$$

In the appendix we prove that $\widetilde{B}$ equipped with the norm $\|f\|_{\widetilde{B}}=\sup _{x \in \mathbb{R}^{3}, k \in I}|f(x, k)|$ is a Banach space (see Lemma A.1) and that for $f \in \widetilde{B}$ the operators

$$
\begin{aligned}
(\widetilde{T} f)(x, k) & :=-\frac{1}{2 \pi} \int \frac{e^{-i|k||x-y|}}{\langle x\rangle^{s}|x-y|}\langle y\rangle^{s} V(y) f(y, k) d y \\
\left(\widetilde{T}^{\prime} f\right)(x, k) & :=\frac{i}{2 \pi} \frac{k_{l}}{|k|} \int \frac{e^{-i|k||x-y|}}{\langle x\rangle^{s}}\langle y\rangle^{s} V(y) f(y, k) d y, \quad \text { and } \\
(\widetilde{K} f)(x, k) & :=\int_{k_{l}^{0}}^{k_{l}} f\left(x,\left(k_{l}^{\prime}, k_{\bar{l}}\right)\right) d k_{l}^{\prime}
\end{aligned}
$$

belong to $\mathcal{L}(\widetilde{B})$ if $s>0$ is chosen such that $\langle x\rangle^{s} V(x)$ still satisfies (V) 3 (see Lemma A.2). 
Noting that $\langle x\rangle^{-s} \partial_{k_{l}} g(x, k)$ and $\langle x\rangle^{-s} \eta\left(x,\left(k_{l}^{0}, k_{l}\right)\right)$ belong to $\widetilde{B}$ (see Lemma A.2), equation (38) can be written as

$$
\tilde{\xi}=\langle\cdot\rangle^{-s} \partial_{k_{l}} g+\tilde{T}^{\prime} \tilde{K} \tilde{\xi}+\tilde{T}^{\prime}\langle\cdot\rangle^{-s} \eta\left(\cdot,\left(k_{l}^{0}, k_{\bar{l}}\right)\right)+\tilde{T} \tilde{\xi}
$$

where $\langle\cdot\rangle^{-s}$ denotes the operator of multiplication with $\langle x\rangle^{-s}$ in $\widetilde{B}$. To prove that this equation has a unique solution in $\widetilde{B}$ we show that $(1-\widetilde{T})^{-1} \in \mathcal{L}(\widetilde{B})$ exists and that

$$
\tilde{\xi}=(1-\tilde{T})^{-1}\left(\langle\cdot\rangle^{-s} \partial_{k_{l}} g+\tilde{T}^{\prime}\langle\cdot\rangle^{-s} \eta\left(\cdot,\left(k_{l}^{0}, k_{l}\right)\right)\right)+(1-\tilde{T})^{-1} \tilde{T}^{\prime} \tilde{K} \tilde{\xi}
$$

has a unique solution. The former will be content of Lemma 3.4, and to see the latter note that according to Lemma A.2.(i)

$$
\left\|(1-\widetilde{T})^{-1} \widetilde{T}^{\prime} \widetilde{K}\right\|_{\mathcal{L}(\widetilde{B})} \leq\left\|(1-\widetilde{T})^{-1} \widetilde{T}^{\prime}\right\|_{\mathcal{L}(\widetilde{B})} 2 \delta_{l}
$$

Also $\left\|(1-\tilde{T})^{-1}\right\|_{\mathcal{L}(\widetilde{B})}$ and $\left\|\widetilde{T}^{\prime}\right\|_{\mathcal{L}(\widetilde{B})}$ depend on $\delta_{l}$ since the space $\widetilde{B}$ itself depends on $\delta_{l}$. But the norm of these operators decreases as $\delta_{l}$ decreases since according to Lemma A.1.(ii) and the constructions in the proofs of Lemma A.2 and Lemma $3.4\left\|\widetilde{T}^{\prime}\right\|_{\mathcal{L}(\widetilde{B})} \leq \sup _{k \in I}\left\|T_{k}^{\prime s}\right\|_{\mathcal{L}(B)}$ and $\left\|(1-\widetilde{T})^{-1}\right\|_{\mathcal{L}(\widetilde{B})} \leq$ $\sup _{k \in I}\left\|\left(1-T_{k}^{s}\right)^{-1}\right\|_{\mathcal{L}(B)}$. Thus one can choose $\delta_{l}$ such that

$$
\left\|(1-\widetilde{T})^{-1} \widetilde{T}^{\prime} \widetilde{K}\right\|_{\mathcal{L}(\widetilde{B})}<1
$$

Then (39) has a unique solution $\tilde{\xi} \in \widetilde{B}$ since $(1-\widetilde{T})^{-1} \widetilde{T}^{\prime} \widetilde{K}$ is a contraction in a complete metric space.

Now $\xi(x, k)=\langle x\rangle^{s} \tilde{\xi}(x, k)$ is a solution of $(36)$ and $f(x, k)=\eta\left(x,\left(k_{l}^{0}, k_{\bar{l}}\right)\right)+\int_{k_{l}^{0}}^{k_{l}} \xi\left(x,\left(k_{l}^{\prime}, k_{\bar{l}}\right)\right) d k_{l}^{\prime}$ is a solution of (31). Recall that to conclude $f(x, k)=\eta(x, k)$, i.e. that $\xi$ is the partial derivative of $\eta$ with respect to $k_{l}$, we need to show $f(\cdot, k) \in B$. By construction $\left.\sup _{k \in I} \mid \xi(x, k)\right) \mid=O\left(|x|^{s}\right)$ for $|x| \rightarrow \infty$ and therefore also $|f(x, k)|=O\left(|x|^{s}\right)$ for any $k \in I$. Thus writing $V(x) f(x, k)=\langle x\rangle^{s} V(x)\langle x\rangle^{-s} f(x, k)$ and observing that $\langle x\rangle^{s} V(x)$ satisfies $(\mathbf{V})_{\mathbf{3}}$ and $\langle x\rangle^{-s} f(x, k)$ is bounded we use Lemma 3.3. (ii) to conclude that $f(\cdot, k) \in B$.

To complete the proof of part (i) for $|\alpha|=1$ we need to show the following lemma:

3.4 Lemma. $(1-\widetilde{T})^{-1} \in \mathcal{L}(\widetilde{B})$ exists.

Proof. First we show that $\left(1-T_{k}^{s}\right)^{-1} \in \mathcal{L}(B)$ exists, where $T_{k}^{s}:=\langle\cdot\rangle^{-s} T_{k}\langle\cdot\rangle^{s} \in \mathcal{L}(B)$. Since $\langle x\rangle^{s} V(x)$ meets the requirements of Lemma 3.3 and multiplication by $\langle x\rangle^{-s}$ is a bounded operation on $B$, $T_{k}^{s}$ is compact. Therefore $\left(1-T_{k}^{s}\right)^{-1}$ exists if the homogeneous equation $\left(1-T_{k}^{s}\right) f_{s}=0$ has only the trivial solution $f_{s}=0$. Now let $f_{s} \in B$ be a solution of the homogeneous equation which explicitly reads

$$
f_{s}(x)=\left(T_{k}^{s} f_{s}\right)(x)=-\frac{1}{2 \pi} \int \frac{e^{-i|k||x-y|}}{\langle x\rangle^{s}|x-y|} V(y)\langle y\rangle^{s} f_{s}(y) d y .
$$

Then $f(x):=\langle x\rangle^{s} f_{s}(x)$ is a solution of

$$
f(x)=-\frac{1}{2 \pi} \int \frac{e^{-i|k||x-y|}}{|x-y|} V(y) f(y) d y
$$


and Lemma 3.3.(ii) implies $f \in B$ since $\langle x\rangle^{s} V(x)$ satisfies $(\mathbf{V})_{\mathbf{3}}$ and $\langle x\rangle^{-s} f(x)$ is bounded. Using Lemma 3.3.(iv) we conclude that $f(x)=f_{s}(x)=0$ for $k \neq 0$. Therefore $\left(1-T_{k}^{s}\right)^{-1}$ exists for any $k \in I$. The continuity of $\left(1-T_{k}^{s}\right)^{-1}$ with respect to $k$ follows again from the continuity of $\left(1-T_{k}^{s}\right)$.

Using Lemma A.1.(ii) we define the operator $(1-\tilde{T})^{-1} \in \mathcal{L}(\widetilde{B})$. Since $\left(1-T_{k}^{s}\right)\left(1-T_{k}^{s}\right)^{-1}=(1-$ $\left.T_{k}^{s}\right)^{-1}\left(1-T_{k}^{s}\right)=1$ holds for all $k \in I$, also $(1-\tilde{T})(1-\widetilde{T})^{-1}=(1-\widetilde{T})^{-1}(1-\widetilde{T})=1$ holds.

It is now easy to prove the existence of higher order derivatives by induction. From the proof for $|\alpha|=1$ we conclude that if $\eta(x, k) \in B$ is a solution of $(31)$ then $\langle x\rangle^{-s} \partial_{k_{l}} \eta(x, k)$ is given by the unique solution $\xi(x, k)$ in $B$ of

$$
\begin{aligned}
\xi(x, k)= & \langle x\rangle^{-s} \partial_{k_{l}} g(x, k)+\frac{i}{2 \pi} \frac{k_{l}}{|k|} \int \frac{e^{-i|k||x-y|}}{\langle x\rangle^{s}} V(y) \eta(y, k) d y \\
& -\frac{1}{2 \pi} \int \frac{e^{-i|k||x-y|}}{\langle x\rangle^{s}|x-y|}\langle y\rangle^{s} V(y) \xi(y, k) d y
\end{aligned}
$$

for any $k \in \mathbb{R}^{3} \backslash\{0\}$. In general, assume that $\eta(x, \cdot) \in C^{p}\left(\mathbb{R}^{3} \backslash\{0\}\right)$ for some $p<n-2$ and that $\langle x\rangle^{-s-p+1} \partial_{k}^{\alpha} \eta(x, k),|\alpha|=p$, is given by the unique solution $\xi(x, k)$ of

$$
\begin{aligned}
\xi(x, k)= & \langle x\rangle^{-s-p+1}\left[\partial_{k}^{\alpha} g(x, k)+\partial_{k}^{\alpha}\left(T_{k} \eta\right)(x, k)-\left(T_{k} \partial_{k}^{\alpha} \eta\right)(x, k)\right] \\
& +\left(T_{k}^{s+p-1} \xi\right)(x, k)
\end{aligned}
$$

in $B$, where $T_{k}^{s+p-1}$ is given by

$$
\left(T_{k}^{s+p-1} f\right)(x):=-\frac{1}{2 \pi} \int \frac{e^{-i|k||x-y|}}{\langle x\rangle^{s+p-1}|x-y|} V(y)\langle y\rangle^{s+p-1} f(y) d y .
$$

Then one can prove by exactly the same method as in the case $|\alpha|=1$ that $\partial_{k_{l}} \xi(x, k)$ exists: Equation (40) is analogous to (33) where $g$ is replaced by $\langle x\rangle^{-s-p+1}\left[\partial_{k}^{\alpha} g(x, k)+\partial_{k}^{\alpha}\left(T_{k} \eta\right)(x, k)-\left(T_{k} \partial_{k}^{\alpha} \eta\right)(x, k)\right] \in B$ and $T_{k}$ by $T_{k}^{s+p-1}$. As long as $\langle y\rangle^{s+p-1} V(y)$ satisfies $(\mathbf{V})_{3}$ the proof of differentiability of the solution of (40) can be done along the same lines as for $|\alpha|=1$.

Proof [of part (ii) of Theorem 3.1]. From the continuity of $\Phi$ and the fact that $\lim _{|x| \rightarrow \infty}(\Phi(x, k)-$ $\left.e^{i k \cdot x}\right)=0$ for all $k \neq 0$ Ikebe already concluded that for compact $D \subset \mathbb{R}^{3} \backslash\{0\}$

$$
\sup _{x \in \mathbb{R}^{3}, k \in D}|\Phi(x, k)|<\infty
$$

holds. It remains to examine the cases $k \rightarrow 0$ and $|k| \rightarrow \infty$. If $H$ has a zero-energy resonance or eigenvalue, according to Jensen and Kato, ${ }^{14}$ the spectral density is singular at $E=0$. Since the spectral density and the generalized eigenfunctions are closely related, ${ }^{20}$ we expect that in this case also the generalized eigenfunctions become singular at $k=0$.

But assuming that $H$ has neither a resonance nor an eigenvalue at $E=0$, the eigenfunctions are uniformly bounded near $k=0$ :

3.5 Proposition. Let the potential satisfy $(\mathbf{V})_{\mathbf{n}}$ for some $n \geq 3$. If $H$ has no zero-energy resonance or eigenvalue, then for any compact $D \subset \mathbb{R}^{3}$ 


$$
\sup _{x \in \mathbb{R}^{3}, k \in D}|\Phi(x, k)|<\infty
$$

and for any $\alpha$ with $|\alpha|<n-2$ there is a $c_{\alpha}<\infty$ such that

$$
\sup _{k \in D \backslash\{0\}}\left|\partial_{k}^{\alpha} \Phi(x, k)\right|<c_{\alpha}(1+|x|)^{|\alpha|} .
$$

Proof. If $H$ has no zero-energy resonance or eigenvalue the homogeneous equation $f=T_{0} f$ has no solution in $B$ since, according to Lemma 3.3 , under the conditions $(\mathbf{V})_{3}$ any solution of $f=T_{0} f$ is a solution of $\left(-\frac{1}{2} \Delta+V(x)\right) f(x)=0$ with $f(x)=O\left(|x|^{-1}\right)$. And a solution $f \in B$ of $H f=0$ with $f(x)=O\left(|x|^{-1}\right)$ is in particular a resonance. Thus either $f \in L^{2}$, i.e.zero is an eigenvalue, or $f \notin L^{2}$, but then zero is a resonance.

Thus $\left(1-T_{k}\right)^{-1}$ exists for all $k \in D$ and, recalling $\eta(x, k)=\left(\left(1-T_{k}\right)^{-1} g\right)(x, k)$,

$$
\sup _{x \in \mathbb{R}^{3}, k \in D}|\eta(x, k)|=\sup _{k \in D}\left\|\eta_{k}\right\|_{B} \leq \sup _{k \in D}\left\|\left(1-T_{k}\right)^{-1}\right\|{ }_{\mathcal{L}(B)}\left\|g_{k}\right\|_{B}<\infty,
$$

since $\left\|\left(1-T_{k}\right)^{-1}\right\|_{\mathcal{L}(B)}$ is a continuous function on a compact set and therefore bounded. Recalling $\Phi(x, k)=e^{i k \cdot x}+\eta(x, k)$ the proof of the first statement is complete.

The bounds for the partial derivatives near zero also follow from the fact that $\left(1-T_{0}\right)^{-1}$ exists if zero is neither a resonance nor an eigenvalue of $H$. To see this we introduce spherical coordinates $(|k|, \omega)$, $|k| \in(0, \infty)$ and $\omega \in S^{2}$ for $k$. If we replace $\partial_{k_{i}}|k|=k_{i} /|k|=\omega \cdot e_{i}$ in Equation (40), it has a unique solution $\xi(\cdot,|k|, \omega) \in B$ also for $|k|=0$. Thus $\lim _{|k| \rightarrow 0} \partial_{k}^{\alpha} \eta(x,|k|, \omega)$ exists for all $\omega \in S^{2}$. As in the first part of this proof

$$
\sup _{x \in \mathbb{R}^{3}, k \in D \backslash\{0\}} \frac{\partial_{k}^{\alpha} \eta(x, k)}{\langle x\rangle^{s+|\alpha|-1}} \leq \sup _{x \in \mathbb{R}^{3},|k| \in[0, R], \omega \in S^{2}}|\xi(x,|k|, \omega)|<\infty,
$$

for some $R$ such that $D \subset K_{R}$, follows from the fact that $\xi(\cdot,|k|, \omega) \in B$ depends continuously on $k$. Noting $\left|\partial_{k}^{\alpha} e^{i k \cdot x}\right|=\left|x_{1}^{\alpha_{1}} x_{2}^{\alpha_{2}} x_{3}^{\alpha_{3}} e^{i k \cdot x}\right|<\langle x\rangle^{|\alpha|}$ completes the proof.

To prove the uniform bound on $\Phi$ and its derivatives it remains to examine their behavior for large $k$. This can be done using the Born series. As expected on physical grounds the generalized eigenfunctions for large momentum are essentially plane waves:

3.6 Proposition. Let the potential $V$ satisfy $(\mathbf{V})_{\mathbf{n}}$ for some $n \geq 3$. Then

$$
\lim _{|k| \rightarrow \infty} \sup _{x \in \mathbb{R}^{3}}\langle x\rangle^{-|\alpha|}\left|\partial_{k}^{\alpha} \Phi(x, k)-\partial_{k}^{\alpha} e^{i k \cdot x}\right|=0
$$

for every $|\alpha| \leq n-2$.

Proof. First we will show that the function $\eta(x, k)=\Phi(x, k)-e^{i k \cdot x}$ converges uniformly to zero for $|k| \rightarrow \infty$. Recall (34): 


$$
\eta(\cdot, k)=\left(1-T_{k}\right)^{-1} g(\cdot, k) .
$$

We shall show that $\lim _{|k| \rightarrow \infty}\|g(\cdot, k)\|_{B}=0$, but we have no simple control of the norm of $\left(1-T_{k}\right)^{-1}$, for example in terms of the Born series, since

$$
\left\|T_{k}\right\|_{\mathcal{L}(B)}=\sup _{x \in \mathbb{R}^{3}} \int \frac{|V(y)|}{|x-y|} d y=\text { const. }
$$

does not depend on $|k|$. Following Zemach and Klein ${ }^{21}$ we iterate Equation (33) once and obtain

$$
\eta(\cdot, k)=g(\cdot, k)+T_{k} g(\cdot, k)+T_{k}^{2} \eta(\cdot, k),
$$

with the formal solution

$$
\eta(\cdot, k)=\left(1-T_{k}^{2}\right)^{-1}\left(g(\cdot, k)+T_{k} g(\cdot, k)\right) .
$$

If equation (41) has a unique solution it must equal the unique solution of (33) since any solution of (33) is clearly also a solution of (41). We shall now first establish that a) $\left(1-T_{k}^{2}\right)^{-1} \rightarrow 1$ for $|k| \rightarrow \infty$ and then that b) $\left\|g(\cdot, k)+T_{k} g(\cdot, k)\right\|_{B} \rightarrow 0$ for $|k| \rightarrow \infty$, since then

$$
\lim _{|k| \rightarrow \infty}\|\eta(\cdot, k)\|_{B} \leq \lim _{|k| \rightarrow \infty}\left\|\left(1-T_{k}^{2}\right)^{-1}\right\|_{\mathcal{L}(B)} \|\left(g(\cdot, k)+T_{k} g(\cdot, k) \|_{B}=0 .\right.
$$

a) follows from

3.7 Lemma. Let $V \in L^{1} \cap L^{2}$ then

$$
\lim _{|k| \rightarrow \infty}\left\|T_{k}^{2}\right\|_{\mathcal{L}(B)}=0
$$

(43) also holds, if $T_{k}^{2}$ is understood as an operator on bounded continuous functions.

Now $|k|$ can be chosen such that $\left\|T_{k}^{2}\right\|_{\mathcal{L}(B)}<1$ and then $\left(1-T_{k}^{2}\right)^{-1}$ is given as the norm convergent Born series:

$$
\left(1-T_{k}^{2}\right)^{-1}=\sum_{n=0}^{\infty}\left(T_{k}^{2}\right)^{n}
$$

Thus $\lim _{|k| \rightarrow \infty}\left\|\left(1-T_{k}^{2}\right)^{-1}-1\right\|_{\mathcal{L}(B)}=0$.

Proof [of Lemma 3.7]. We compute for $f \in B$

$$
\begin{aligned}
\left(T_{k}^{2} f\right)(x) & =\frac{1}{4 \pi^{2}} \int \frac{e^{-i|k||x-y|}}{|x-y|} V(y) \int \frac{e^{-i|k||y-z|}}{|y-z|} V(z) f(z) d z d y \\
& =\frac{1}{4 \pi^{2}} \int V(z) f(z) \int \frac{e^{-i|k|(|x-y|+|y-z|)}}{|x-y||y-z|} V(y) d y d z \\
& =\frac{1}{4 \pi^{2}} \int \frac{V(z)}{|x-z|} f(z)\left[|x-z| \int \frac{e^{-i|k|(|x-y|+|y-z|)}}{|x-y||y-z|} V(y) d y\right] d z \\
& =: \frac{1}{4 \pi^{2}} \int \frac{V(z)}{|x-z|} f(z) I^{V}(x, z,|k|) d z .
\end{aligned}
$$


Zemach and Klein ${ }^{21}$ showed that for $V \in C_{0}^{1}\left(\mathbb{R}^{3}\right)$

$$
\lim _{|k| \rightarrow \infty} \sup _{x, z \in \mathbb{R}^{3}}\left|I^{V}(x, z,|k|)\right|=0,
$$

i.e. that $\lim _{|k| \rightarrow \infty}\left\|T_{k}^{2}\right\|_{\mathcal{L}(B)}=0$ holds for $V \in C_{0}^{1}\left(\mathbb{R}^{3}\right)$.

Potentials $V \in L^{1} \cap L^{2}$ will be approximated in the following norm:

$$
\||V|||=\sup _{x \in \mathbb{R}^{3}} \int \frac{|V(y)|}{|x-y|} d y .
$$

Observing that

$$
\begin{aligned}
\|V\| \| & \leq \sup _{x \in \mathbb{R}^{3}} \int_{|x-y|<1} \frac{V(y)}{|x-y|} d y+\sup _{x \in \mathbb{R}^{3}} \int_{|x-y| \geq 1} \frac{V(y)}{|x-y|} d y \\
& \leq c \quad\left(\|V\|_{L^{2}}+\|V\|_{L^{1}}\right)<\infty,
\end{aligned}
$$

where we used Schwarz's inequality for the $\|V\|_{L^{2}}$ term, we conclude that, since any $V \in L^{1} \cap L^{2}$ can be approximated by a function $U \in C_{0}^{1}$ simultaneously in $L^{1}$ and $L^{2}$-norm, this is also true for the ||| |||-norm. Thus we get the following bound for the norm of $T_{k}^{2}$ :

$$
\begin{aligned}
\left\|T_{k}^{2} f\right\|_{B}= & \sup _{x \in \mathbb{R}^{3}} \mid \frac{1}{(2 \pi)^{2}} \int \frac{e^{-i|k||x-y|}}{|x-y|}(V(y)-U(y)+U(y)) \\
& \times \int \frac{e^{-i|k||y-z|}}{|y-z|} V(z) f(z) d z d y \mid \\
\leq & \|f\|_{B}\left|\left\|V|\||\left(|| V-U\left|\|+\sup _{x, z \in \mathbb{R}^{3}}\right| I^{U}(x, z,|k|) \mid\right) .\right.\right.
\end{aligned}
$$

The first term in the brackets becomes small for appropriately chosen $U \in C_{0}^{1}$ while the second one converges to zero for $|k| \rightarrow \infty$.

We now proceed to b) namely that $\lim _{|k| \rightarrow \infty}\left\|g(\cdot, k)+T_{k} g(\cdot, k)\right\|_{B}=0$. By $T_{k} g(x, k)=T_{k}^{2} e^{i k \cdot x}$ and Lemma $3.7 \lim _{|k| \rightarrow \infty}\left\|T_{k} g(\cdot, k)\right\|_{B}=0$ follows immediately. To get the analogous statement for $g(\cdot, k)$ we assume first again $V \in C_{0}^{1}\left(\mathbb{R}^{3}\right)$. Then

$$
\begin{aligned}
|g(x, k)|= & \frac{1}{2 \pi}\left|\int \frac{e^{-i|k||x-y|}}{|x-y|} V(y) e^{-i k \cdot(x-y)} d y\right| \\
\leq & \frac{1}{2 \pi}\left|\int_{\theta<\theta_{0}} \frac{e^{-i|k||z|(1+\cos \theta)}}{|z|} V(x-z) d z\right| \\
& +\frac{1}{2 \pi} \int_{\theta \geq \theta_{0}} \frac{|V(x-z)|}{|z|} d z
\end{aligned}
$$


holds. Herein $\theta$ denotes the angle between $z=(x-y)$ and $k$. Stationary phase methods on the first term yields

$$
\begin{array}{r}
\frac{1}{2 \pi}\left|\int_{\theta<\theta_{0}} \frac{1}{i|k|(1+\cos \theta)}\left(\frac{d}{d|z|} e^{-i|k||z|(1+\cos \theta)}\right) V(x-z)\right| z|d| z|d \Omega(z)| \\
=\frac{1}{2 \pi}\left|\int_{\theta<\theta_{0}} \frac{1}{i|k|(1+\cos \theta)} e^{-i|k||z|(1+\cos \theta)}\left(\frac{d}{d|z|} V(x-z)|z|\right) d\right| z|d \Omega(z)| \\
\leq \frac{1}{2 \pi} \frac{1}{|k|\left(1+\cos \theta_{0}\right)} \int\left|\frac{d}{d|z|} V(x-z)\right| z|| d|z| d \Omega(z) \\
\leq \frac{c}{|k|\left(1+\cos \theta_{0}\right)} \stackrel{|k| \rightarrow \infty}{\longrightarrow} 0,
\end{array}
$$

where

$$
\sup _{x \in \mathbb{R}^{3}} \int\left|\frac{\frac{d}{d|z|} V(x-z)|z|}{|z|^{2}}\right| d z=c<\infty
$$

was used. This follows directly from $V \in C_{0}^{1}$.

The second term in (45) is an integration over a cone with opening angle $\theta_{0}$ where the potential in the integrand has compact support, is bounded and displaced by $-x$. Thus

$$
\lim _{\theta_{0} \rightarrow \pi} \sup _{x \in \mathbb{R}^{3}} \int_{\theta \geq \theta_{0}} \frac{|V(x-z)|}{|z|} d z \leq c \lim _{\theta_{0} \rightarrow \pi} \int_{\theta_{0}}^{\pi}|\sin \theta| d \theta=0
$$

and $\lim _{|k| \rightarrow \infty} \sup _{x \in \mathbb{R}^{3}}|g(x, k)|=0$ follows. To get this for $V \in L^{1} \cap L^{2}$ we approximate $V$ by $U \in C_{0}^{1}$ as in Lemma 3.7.

Analogously we show that $\langle x\rangle^{-|\alpha|}\left|\partial_{k}^{\alpha} \Phi(x, k)-\partial_{k}^{\alpha} e^{i k \cdot x}\right|$ vanish uniformly for $|k| \rightarrow \infty$. According to the proof of part (i) of Theorem $3.1 \partial_{k}^{\alpha} \eta(x, k)$ is obtained as the unique solution of (40) in $B$. For large $|k|$ the operator $\left(1-\left(T_{k}^{s+p-1}\right)^{2}\right)^{-1}$ with $p=|\alpha|$ can be expanded in terms of the Born series and the solution of the modified equation is given by

$$
\begin{aligned}
\xi= & \left(1-\left(T_{k}^{s+p-1}\right)^{2}\right)^{-1}\left(\langle\cdot\rangle^{-s-p+1}\left[\partial_{k}^{\alpha} g+\partial_{k}^{\alpha} T_{k} \eta-T_{k} \partial_{k}^{\alpha} \eta\right]\right. \\
& \left.+T_{k}^{s+p-1}\langle\cdot\rangle^{-s-p+1}\left[\partial_{k}^{\alpha} g+\partial_{k}^{\alpha} T_{k} \eta-T_{k} \partial_{k}^{\alpha} \eta\right]\right) .
\end{aligned}
$$

It can be shown by the same methods as in the case of $g(x, k)$ that the term, on which $\left(1-\left(T_{k}^{s+p-1}\right)^{2}\right)^{-1}$ acts, uniformly approaches zero for $|k| \rightarrow \infty$, which completes the proof of the proposition.

The uniform boundedness of $\Phi$ as well as the bounds on its partial derivatives with respect to $k$ now follow from Proposition 3.5 and Proposition 3.6.

We will end this section with two corollaries to Theorem 3.1 and Proposition 3.6: The first one states that the Riemann-Lebesgue lemma holds also for the generalized Fourier transformation and its inverse. Furthermore, the differentiability of the generalized Fourier transform of a function is connected to its decay as in the case of the ordinary Fourier transform. Related results can be found in a work by Isozaki. ${ }^{22}$ 
3.8 Corollary. Let $V$ satisfy $(\mathbf{V})_{\mathbf{n}}$ with some $n \geq 3$ and let zero not be an eigenvalue or resonance of $H$. Then, for any $N \leq n-2$ and any $f$ such that $\langle x\rangle^{N} f \in L^{1}\left(\mathbb{R}^{3}\right) \mathcal{F}_{ \pm} f$ and $\mathcal{F}_{ \pm}^{-1} f$ are in $C^{N}\left(\mathbb{R}^{3}\right)$ and $\partial_{k}^{\alpha} \mathcal{F}_{ \pm} f \in C_{\infty}\left(\mathbb{R}^{3}\right)$ and $\partial_{k}^{\alpha} \mathcal{F}_{ \pm}^{-1} f \in C_{\infty}\left(\mathbb{R}^{3}\right)$ for all $\alpha$ with $|\alpha| \leq N$.

Proof. Let $\langle x\rangle^{N} f \in L^{1}, 0 \leq|\alpha| \leq N$, then e.g.

$$
\begin{aligned}
\partial_{k}^{\alpha}\left(\mathcal{F}_{+} f\right)(k) & =\partial_{k}^{\alpha} \frac{1}{(2 \pi)^{\frac{3}{2}}} \int \Phi_{+}^{*}(x, k) f(x) d x=\frac{1}{(2 \pi)^{\frac{3}{2}}} \int \partial_{k}^{\alpha} \Phi_{+}^{*}(x, k) f(x) d x \\
& =\frac{1}{(2 \pi)^{\frac{3}{2}}} \int \partial_{k}^{\alpha} e^{-i k \cdot x} f(x) d x+\frac{1}{(2 \pi)^{\frac{3}{2}}} \int \partial_{k}^{\alpha} \eta_{+}^{*}(x, k) f(x) d x
\end{aligned}
$$

is bounded and continuous since $\left|\partial_{k}^{\alpha} \Phi_{+}^{*}(x, k)\right|$ is bounded by $c_{\alpha}\langle x\rangle^{|\alpha|}$ according to Theorem 3.1 and $\langle x\rangle^{|\alpha|} f \in L^{1}$. Furthermore, the first term in the second line belongs to $C_{\infty}$ by the ordinary RiemannLebesgue lemma and the second term belongs to $C_{\infty}$ since $\langle x\rangle^{-|\alpha|}\left|\partial_{k}^{\alpha} \eta_{+}^{*}(x, k)\right|$ tends uniformly to zero for $|k| \rightarrow \infty$ according to Proposition 3.6.

The second corollary concerns the so called T-matrix, an object widely discussed in quantum mechanical scattering theory. Let $V$ satisfy $(\mathbf{V})_{\mathbf{3}}$, then the T-matrix $\mathbf{T}(\cdot, \cdot)$ is defined by

$$
\mathbf{T}\left(k, k^{\prime}\right)=(2 \pi)^{-3} \int e^{-i k \cdot x} V(x) \Phi_{-}\left(x, k^{\prime}\right) d x .
$$

There are several results about the analyticity of the T-matrix for potentials with exponential decay. ${ }^{23}$ The following corollary gives sufficient conditions for $\mathbf{T}\left(k, k^{\prime}\right)$ to be continuously differentiable.

3.9 Corollary. Let $V$ satisfy $(\mathbf{V})_{\mathbf{n}}$ for some $n \geq 3$ and let zero be neither a resonance nor an eigenvalue of $H$. Then

(i) $\mathbf{T}(\cdot, \cdot) \in C^{n-3}\left(\mathbb{R}^{3} \times\left(\mathbb{R}^{3} \backslash\{0\}\right)\right)$

(ii) For every multi-index $\alpha$ with $|\alpha| \leq n-3$

$$
\sup _{k \in \mathbb{R}^{3}, k^{\prime} \in \mathbb{R}^{3} \backslash\{0\}}\left|\partial_{k^{\prime}}^{\alpha} \mathbf{T}\left(k, k^{\prime}\right)\right|<\infty .
$$

Acknowledgments. This work was supported by DFG. We thank W. Amrein for very helpful correspondence, for reference 14 and for sending us his preprints 7 and 8 prior to publication. Very helpful discussions with S. Goldstein, A. Soffer and N. Zanghì are gratefully acknowledged. 


\section{A Appendix}

In this appendix we prove two lemmas used in Section 3.

\section{A.1 Lemma.}

(i) The space $\widetilde{B}$ equipped with the norm

$$
\|f\|_{\widetilde{B}}=\sup _{x \in \mathbb{R}^{3}, k \in I}|f(x, k)|
$$

is a Banach space.

(ii) Let $\left\{A_{k}\right\}_{k \in I} \subset \mathcal{L}(B)$ be a family of bounded operators on $B$ such that $A_{k}$ depends continuously on $k$ with respect to the operator-norm. Then

$$
(A f)(x, k):=\left(A_{k} f(\cdot, k)\right)(x)
$$

defines an operator $A \in \mathcal{L}(\widetilde{B})$ and $\|A\|_{\mathcal{L}(\widetilde{B})} \leq \sup _{k \in I}\left\|A_{k}\right\|_{\mathcal{L}(B)}$.

Proof [of part (i)]. Let $\left\{f_{n}\right\}_{n \in \mathbb{N}}$ be a Cauchy sequence in $\widetilde{B} \subset C\left(\mathbb{R}^{3} \times I\right)$. Then there exists $f \in C\left(\mathbb{R}^{3} \times I\right)$ such that $\lim _{n \rightarrow \infty}\left\|f-f_{n}\right\|_{\widetilde{B}}=0$. It remains to show that $f \in \widetilde{B}$, i.e.that $\lim _{|x| \rightarrow \infty} \sup _{k \in I}|f(x, k)|=0$. But

$$
\begin{aligned}
\sup _{k \in I}|f(x, k)| & \leq \sup _{k \in I}\left|f(x, k)-f_{n}(x, k)\right|+\sup _{k \in I}\left|f_{n}(x, k)\right| \\
& \leq\left\|f-f_{n}\right\|_{\widetilde{B}}+\sup _{k \in I}\left|f_{n}(x, k)\right| .
\end{aligned}
$$

The first term can be made arbitrarily small by appropriately choosing $n$ and the second term vanishes for $|x| \rightarrow \infty$.

Proof [of part (ii)]. Let $f \in \widetilde{B}$. Then for any fixed $k \in I, f(\cdot, k) \in B$ and therefore $A_{k} f(\cdot, k) \in B$. First we show that $A f(\cdot, \cdot) \in C\left(\mathbb{R}^{3} \times I\right)$ :

$$
\begin{aligned}
\left|(A f)(x, k)-(A f)\left(x^{\prime}, k^{\prime}\right)\right| \leq & \left|\left(A_{k} f(\cdot, k)\right)(x)-\left(A_{k^{\prime}} f\left(\cdot, k^{\prime}\right)\right)\left(x^{\prime}\right)\right| \\
\leq & \left|\left(A_{k} f(\cdot, k)\right)(x)-\left(A_{k} f(\cdot, k)\right)\left(x^{\prime}\right)\right| \\
& +\left|\left(\left(A_{k}-A_{k^{\prime}}\right) f(\cdot, k)\right)\left(x^{\prime}\right)\right| \\
& +\left|\left(A_{k^{\prime}}\left(f(\cdot, k)-f\left(\cdot, k^{\prime}\right)\right)\right)\left(x^{\prime}\right)\right|
\end{aligned}
$$

Since $A_{k} f(\cdot, k) \in B$ the first term can be made arbitrary small by choosing $\left|x-x^{\prime}\right|$ small enough. The second term becomes small uniformly in $x^{\prime}$ by choosing $\left|k-k^{\prime}\right|$ small enough since

$$
\sup _{x^{\prime} \in \mathbb{R}^{3}}\left|\left(\left(A_{k}-A_{k^{\prime}}\right) f(\cdot, k)\right)\left(x^{\prime}\right)\right|=\left\|\left(A_{k}-A_{k^{\prime}}\right) f(\cdot, k)\right\|_{B} \leq\left\|A_{k}-A_{k^{\prime}}\right\|_{\mathcal{L}(B)}\|f\|_{\widetilde{B}}
$$

and $A_{k}$ depends continuously on $k$. The third term in (48) yields 


$$
\begin{aligned}
& \sup _{x^{\prime} \in \mathbb{R}^{3}}\left|\left(A_{k^{\prime}}\left(f(\cdot, k)-f\left(\cdot, k^{\prime}\right)\right)\right)\left(x^{\prime}\right)\right| \leq\left\|A_{k^{\prime}}\left|\mathcal{L}_{\mathcal{L}(B)}\right| \mid f(\cdot, k)-f\left(\cdot, k^{\prime}\right)\right\|_{B} \\
& \quad \leq c \max \left(\sup _{\left|x^{\prime}\right|>R}\left|f\left(x^{\prime}, k\right)-f\left(x^{\prime}, k^{\prime}\right)\right|, \sup _{\left|x^{\prime}\right| \leq R}\left|f\left(x^{\prime}, k\right)-f\left(x^{\prime}, k^{\prime}\right)\right|\right),
\end{aligned}
$$

where we used $\sup _{k \in I}\left\|A_{k}\right\|_{\mathcal{L}(B)} \leq c<\infty$. This holds because $\left\|A_{k}\right\|_{\mathcal{L}(B)}$ is a continuous function of $k$ on a compact set. The first term in $\max (.$.$) can be made arbitrary small by choosing R$ large since $f \in \widetilde{B}$. The second term vanishes for $\left|k-k^{\prime}\right| \rightarrow 0$ since a continuous function on a compact domain is uniformly continuous.

We now show that

$$
\lim _{|x| \rightarrow \infty} \sup _{k \in I}(A f)(x, k)=\lim _{|x| \rightarrow \infty} \sup _{k \in I}\left(A_{k} f(\cdot, k)\right)(x)=0 .
$$

Suppose that this is wrong, then there exists an $\epsilon>0$ and a sequence $\left\{x_{n}, k_{n}\right\}_{n \in \mathbb{N}} \subset \mathbb{R}^{3} \times I$ with $\lim _{n \rightarrow \infty} x_{n}=\infty$, such that $\left|\left(A_{k_{n}} f\left(\cdot, k_{n}\right)\right)\left(x_{n}\right)\right|>\epsilon \quad \forall n \in \mathbb{N}$. Since $I$ is compact, $\left\{k_{n}\right\}$ contains a convergent subsequence (for simplicity also denoted by $\left\{k_{n}\right\}$ ) with $\lim _{n \rightarrow \infty} k_{n}=k \in I$. Now

$$
\begin{aligned}
\left|A_{k_{n}} f\left(x_{n}, k_{n}\right)\right| \leq & \left|A_{k_{n}}\left(f\left(x_{n}, k_{n}\right)-f\left(x_{n}, k\right)\right)\right|+\left|\left(A_{k_{n}}-A_{k}\right) f\left(x_{n}, k\right)\right| \\
& +\left|A_{k} f\left(x_{n}, k\right)\right|
\end{aligned}
$$

where the first two terms get arbitrary small as $n \rightarrow \infty$ as has just been shown, and the third term gets arbitrary small as $n \rightarrow \infty$ since $A_{k} f(\cdot, k) \in B$. Thus we have a contradiction and $A f \in \widetilde{B}$ follows.

The estimate for the norm follows directly from

$$
\|A f\|_{\widetilde{B}}=\sup _{x \in \mathbb{R}^{3}, k \in I}\left|\left(A_{k} f(\cdot, k)\right)(x)\right| \leq \sup _{k \in I}\left\|A_{k}\right\|_{\mathcal{L}(B)}\|f(\cdot, k)\|_{B} \leq \sup _{k \in I}\left\|A_{k}\right\|_{\mathcal{L}(B)}\|f\|_{\widetilde{B}}
$$

A.2 Lemma. Let $V$ satisfy $(\mathbf{V})_{\mathbf{3}}$ and let $s>0$ such that $\langle x\rangle^{s} V(x)$ still satisfies $(\mathbf{V})_{\mathbf{3}}$. For $f \in \widetilde{B}$ let

$$
\begin{aligned}
(\tilde{T} f)(x, k) & :=-\frac{1}{2 \pi} \int \frac{e^{-i|k||x-y|}}{\langle x\rangle^{s}|x-y|}\langle y\rangle^{s} V(y) f(y, k) d y, \\
\left(\widetilde{T}^{\prime} f\right)(x, k) & :=\frac{i}{2 \pi} \frac{k_{l}}{|k|} \int \frac{e^{-i|k||x-y|}}{\langle x\rangle^{s}}\langle y\rangle^{s} V(y) f(y, k) d y, \quad \text { and } \\
(\tilde{K} f)(x, k) & :=\int_{k_{l}^{0}}^{k_{l}} f\left(x,\left(k_{l}^{\prime}, k_{\bar{l}}\right)\right) d k_{l}^{\prime}
\end{aligned}
$$

then

(i) the operators $\tilde{T}, \tilde{T}^{\prime}$ and $\tilde{K}$ belong to $\mathcal{L}(\widetilde{B})$ and $\|\tilde{K}\|_{\mathcal{L}(\tilde{B})} \leq 2 \delta_{l}$, where $2 \delta_{l}$ is the length of the interval $I_{l}$.

(ii) The functions $\langle x\rangle^{-s} \partial_{k_{l}} g(x, k)$ and $\langle x\rangle^{-s} \eta\left(x,\left(k_{l}^{0}, k_{\bar{l}}\right)\right)$ belong to $\widetilde{B}$. 
Proof [of part (i)]. Let $f \in B$ and define

$$
\begin{aligned}
\left(T_{k}^{s} f\right)(x) & =-\frac{1}{2 \pi} \int \frac{e^{-i|k||x-y|}}{\langle x\rangle^{s}|x-y|}\langle y\rangle^{s} V(y) f(y) d y, \\
\left(T_{k}^{\prime}{ }^{s} f\right)(x) & =\frac{i}{2 \pi} \frac{k_{l}}{|k|} \int \frac{e^{-i|k||x-y|}}{\langle x\rangle^{s}}\langle y\rangle^{s} V(y) f(y) d y,
\end{aligned}
$$

then for $\tilde{f} \in B$ we have $(\tilde{T} \tilde{f})(x, k)=\left(T_{k}^{s} \tilde{f}(\cdot, k)\right)(x)$ and analogously for $\widetilde{T}^{\prime}$. We shall use Lemma A.1.(ii) to prove that $\tilde{T}$ and $\widetilde{T}^{\prime}$ are in $\mathcal{L}(\widetilde{B})$. We have to show that $\left(T_{k}^{s}\right)_{k \in I}$ and $\left(T_{k}^{\prime s}\right)_{k \in I}$ are families of operators in $\mathcal{L}(B)$ continuously depending on $k$.

Now $\langle y\rangle^{s} V(y)$ still satisfies the conditions (V) $\mathbf{3}$. According to Lemma 3.3.(i) and (v) $\langle x\rangle^{s} T_{k}^{s}$ satisfies the conditions of Lemma A.1.(ii). Multiplication by $\langle x\rangle^{-s}$ is a bounded operation in $B$ and thus also $\left(T_{k}^{s}\right)_{k \in I}$ satisfies the conditions of Lemma A.1.(ii). Hence $\tilde{T} \in \mathcal{L}(\widetilde{B})$.

Next consider $T_{k}^{\prime}$. From $\langle y\rangle^{s} V(y) \in L^{1}$ and

$$
\left|\left(T_{k}^{\prime s} f\right)(x)\right| \leq\langle x\rangle^{-s} \frac{1}{2 \pi} \int\left|\langle y\rangle^{s} V(y)\right| d y\|f\|_{B}
$$

$\lim _{|x| \rightarrow \infty}\left|\left(T_{k}^{\prime s} f\right)(x)\right|=0$ follows. With $\left|e^{i \alpha}-e^{i \alpha^{\prime}}\right| \leq\left|\alpha-\alpha^{\prime}\right|$ for $\alpha, \alpha^{\prime} \in \mathbb{R}$ we estimate

$$
\begin{aligned}
& \left|\langle x\rangle^{s}\left(T_{k}^{s} f\right)(x)-\left\langle x^{\prime}\right\rangle^{s}\left(T_{k}^{\prime} s f\right)\left(x^{\prime}\right)\right| \leq \\
& \quad \leq \frac{1}{2 \pi} \int\left|e^{-i|k||x-y|}-e^{-i|k|\left|x^{\prime}-y\right|}\right|\left|\langle y\rangle^{s} V(y) \| f(y)\right| d y \\
& \quad \leq \frac{1}{2 \pi} \int|k|\left\|x-y|-| x^{\prime}-y||\left|\langle y\rangle^{s} V(y)\right| d y\right\| f \|_{B} \\
& \quad \leq\left|x-x^{\prime}\right||k| \frac{1}{2 \pi} \int\left|\langle y\rangle^{s} V(y)\right| d y\|f\|_{B}
\end{aligned}
$$

which proves the continuity of $\langle x\rangle^{s}\left(T_{k}^{s} s\right)(x)$ in $x$ and thus that of $\left(T_{k}^{s} s\right)(x)$ itself. Therefore $T_{k}^{s} \in \mathcal{L}(B)$. It remains to show that $T_{k}^{\prime}$ is norm continuous with respect to $k$ :

$$
\begin{aligned}
& \left\|\left(T_{k}^{\prime s}-T_{k^{\prime}}^{\prime s}\right) f\right\|_{B}= \\
& =\sup _{x \in \mathbb{R}^{3}}\left|\frac{1}{2 \pi} \int \frac{\langle y\rangle^{s} V(y) f(y)}{\langle x\rangle^{s}}\left(\frac{k_{l}}{|k|} e^{-i|k||x-y|}-\frac{k_{l}^{\prime}}{\left|k^{\prime}\right|} e^{-i\left|k^{\prime}\right||x-y|}\right)\right| \\
& \leq \quad \sup _{x \in \mathbb{R}^{3}}\left|\frac{1}{2 \pi} \int \frac{\langle y\rangle^{s} V(y) f(y)}{\langle x\rangle^{s}} e^{-i|k||x-y|}\left(\frac{k_{l}}{|k|}-\frac{k_{l}^{\prime}}{\left|k^{\prime}\right|}\right)\right| \\
& \quad+\sup _{x \in \mathbb{R}^{3}}\left|\frac{1}{2 \pi} \int \frac{\langle y\rangle^{s} V(y) f(y)}{\langle x\rangle^{s}} \frac{k_{l}^{\prime}}{\left|k^{\prime}\right|}\left(e^{-i|k||x-y|}-e^{-i\left|k^{\prime}\right||x-y|}\right)\right| \\
& \leq \quad c\left|\frac{k_{l}}{|k|}-\frac{k_{l}^{\prime}}{\left|k^{\prime}\right|}\right||| f|| B \\
& \quad+\sup _{x \in \mathbb{R}^{3}} \frac{1}{2 \pi} \int \frac{\left|\langle y\rangle^{s} V(y)\right|}{\langle x\rangle^{s}}\left|e^{-i|k||x-y|}-e^{-i\left|k^{\prime}\right||x-y|}\right| d y\|f\|_{B} .
\end{aligned}
$$


Since we can achieve $c\left|\frac{k_{l}}{|k|}-\frac{k_{l}^{\prime}}{\left|k^{\prime}\right|}\right|<\frac{\epsilon}{2}$ for any $\epsilon>0$ by choosing $\left|k-k^{\prime}\right|$ small it remains to show that also

$$
\sup _{x \in \mathbb{R}^{3}} \frac{1}{2 \pi} \int \frac{\left|\langle y\rangle^{s} V(y)\right|}{\langle x\rangle^{s}}\left|e^{-i|k||x-y|}-e^{-i\left|k^{\prime}\right||x-y|}\right| d y<\frac{\epsilon}{2}
$$

for $\left|k-k^{\prime}\right|$ small. Since $\langle x\rangle^{s} V(x) \in L^{1}$ and $\left|e^{-i|k||x-y|}-e^{-i\left|k^{\prime}\right||x-y|}\right| \leq 2$ there exists $R_{1}$ such that

$$
\begin{gathered}
\sup _{|x|>R_{1}} \frac{1}{2 \pi} \int \frac{\left|\langle y\rangle^{s} V(y)\right|}{\langle x\rangle^{s}}\left|e^{-i|k||x-y|}-e^{-i\left|k^{\prime}\right||x-y|}\right| d y \leq \\
\quad \leq \sup _{|x|>R_{1}} \frac{1}{\langle x\rangle^{s}} \frac{1}{2 \pi} \int 2\left|\langle y\rangle^{s} V(y)\right| d y<\frac{\epsilon}{2} .
\end{gathered}
$$

Similarly there is $R_{2}$ such that

$$
\begin{aligned}
& \sup _{x \in \mathbb{R}^{3}} \frac{1}{2 \pi} \int_{y>R_{2}} \frac{\left|\langle y\rangle^{s} V(y)\right|}{\langle x\rangle^{s}}\left|e^{-i|k||x-y|}-e^{-i\left|k^{\prime}\right||x-y|}\right| d y \leq \\
& \quad \leq \sup _{x \in \mathbb{R}^{3}} \frac{1}{\langle x\rangle^{s}} \frac{1}{2 \pi} \int_{y>R_{2}} 2\left|\langle y\rangle^{s} V(y)\right| d y<\frac{\epsilon}{4},
\end{aligned}
$$

holds. Observing that from $|x|<R_{1}$ and $|y|<R_{2}|x-y|<R_{1}+R_{2}$ follows, we obtain for the remaining part

$$
\begin{aligned}
& \sup _{|x|<R_{1}} \frac{1}{2 \pi} \int_{|y|<R_{2}} \frac{\left|\langle y\rangle^{s} V(y)\right|}{\langle x\rangle^{s}}\left|e^{-i|k||x-y|}-e^{-i\left|k^{\prime}\right||x-y|}\right| d y \leq \\
& \quad \leq|| k|-| k^{\prime}|| \frac{1}{2 \pi}\left(R_{1}+R_{2}\right) \int\left|\langle y\rangle^{s} V(y)\right| d y \leq C\left|k-k^{\prime}\right|<\frac{\epsilon}{4}
\end{aligned}
$$

for $\left|k-k^{\prime}\right|$ sufficiently small. Combining these results we get that for any $\epsilon>0$

$$
\left\|\left(T_{k}^{s}{ }^{s}-T_{k^{\prime}}^{\prime s}\right) f\right\|_{B}<\epsilon\|f\|_{B}
$$

for $\left|k-k^{\prime}\right|$ small enough, which proves the norm continuity of $T_{k}^{\prime} s$. Therefore $T_{k}^{\prime} s$ meets the requirements of Lemma A.1.(ii) and we conclude that $\widetilde{T}^{\prime} \in \mathcal{L}(\widetilde{B})$.

Finally consider $\widetilde{K}$. For $f \in \widetilde{B}$ the continuity of $(\widetilde{K} f)(x, k)=\int_{k_{l}^{0}}^{k_{l}} f\left(x,\left(k_{l}^{\prime}, k_{\bar{l}}\right)\right) d k_{l}^{\prime}$ in $x$ and $k$ is clear. Furthermore

$$
\lim _{|x| \rightarrow \infty} \sup _{k \in I}|(\tilde{K} f)(x, k)| \leq \lim _{|x| \rightarrow \infty} 2 \delta_{l} \sup _{k \in I}|f(x, k)|=0
$$

so that $\tilde{K} \in \mathcal{L}(\widetilde{B})$ and

$$
\|\tilde{K} f\|_{\widetilde{B}}=\sup _{x \in \mathbb{R}^{3}, k \in I}\left|\int_{k_{l}^{0}}^{k_{l}} f\left(x,\left(k_{l}^{\prime}, k_{\bar{l}}\right)\right) d k_{l}^{\prime}\right| \leq 2 \delta_{l}\|f\|_{\widetilde{B}} .
$$


Proof [of part (ii)]. Since $\eta(x, k) \in B$ for all $k \neq 0$ and $I$ is compact, $\langle x\rangle^{-s} \eta\left(x,\left(k_{l}^{0}, k_{\bar{l}}\right)\right) \in \widetilde{B}$ is obvious. Observing

$$
\begin{aligned}
\partial_{k_{l}} g(x, k)= & \frac{i}{2 \pi} \frac{k_{l}}{|k|} \int e^{-i|k||x-y|} V(y) e^{i k \cdot y} d y \\
& -\frac{i}{2 \pi} \int \frac{e^{-i|k||x-y|}}{|x-y|} V(y) y_{l} e^{i k \cdot y} d y,
\end{aligned}
$$

$\langle x\rangle^{-s} \partial_{k_{l}} g(x, k) \in \widetilde{B}$ can be shown using the same types of estimates as in the proof of part (i) of this lemma.

\section{References}

${ }^{1}$ Combes, J.-M., Newton, R.G. and Shtokhamer, R.: Scattering into cones and flux across surfaces, Phys. Rev. D 11, 366-372 (1975).

${ }^{2}$ Daumer, M., Dürr, D., Goldstein, S. and Zanghì, N.: On the Flux-Across-Surfaces Theorem, Letters in Mathematical Physics 38, 103-116 (1996).

${ }^{3}$ Dollard, J.D.: Scattering into cones I, Potential scattering, Comm. Math. Phys. 12 193-203 (1969).

${ }^{4}$ See e.g. pp.278 in Amrein, W.O., Jauch, J.M. and Sinha, K.B.: Scattering Theory in Quantum Mechanics, W.A. Benjamin, Inc., Reading, Massachusetts, 1977.

${ }^{5}$ Daumer, M.: Streutheorie aus der Sicht Bohmscher Mechanik, PhD thesis Ludwig-Maximilians-Universität München (1995).

${ }^{6}$ Daumer, M., Dürr, D., Goldstein, S. and Zanghì, N.: On the quantum probability flux through surfaces, Journal of Stat. Phys. Vol. 88, 967-977 (1997)

${ }^{7}$ Amrein, W.O. and Zuleta, J.L.: Flux and scattering into cones in potential scattering, Helv. Phys. Acta 70, 1 (1997).

8 Amrein, W.O. and Pearson D.B.: Flux and scattering into cones for long range and singular potentials, Journal of Physics A, Vol. 30, 5361-5379 (1997).

9 Journé, J.-L., Soffer, A. and Sogge, C.D.: Decay Estimates for Schrödinger Operators, Comm. Pure. Appl. Math. 44 573-604 (1991).

10 Yajima, K.: The $W^{k, p}$-continuity of wave operators for Schrödinger operators, J. Math. Soc. Japan 47 No. 3, 551-581 (1995).

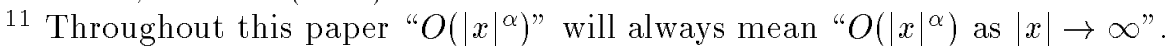

${ }^{12} V: D \rightarrow \mathbb{R}$ is locally Hölder continuous if for every $x \in D$ there is an open neighborhood $U_{x} \subset D$ and an $\alpha_{x}>0, c_{x}>0$ such that $|V(x)-V(y)| \leq c_{x}|x-y|^{\alpha_{x}}$ for all $y \in U_{x}$.

${ }^{13}$ Ikebe, T.: Eigenfunction expansion associated with the Schrödinger operators and their applications to scattering theory, Arch. Rational Mech. Anal. 5, 1-34 (1960).

14 Jensen A. and Kato T.: Spectral properties of Schrödinger operators and time-decay of the wave function, Duke Math. J. 46, 583-611 (1979).

15 l.i.m. $\int$ is a short way of writing $s-\lim _{R \rightarrow \infty} \int_{B_{R}}$ and $s$ - lim denotes the strong limit.

16 Agmon, S.: Spectral Properties of Schrödinger Operators and Scattering Theory, Annali della Scuola Norm. Sup. di Pisa Ser. IV 2, 151-217 (1975). 
${ }^{17}$ We use the usual multi-index notation: $\alpha=\left(\alpha_{1}, \alpha_{2}, \alpha_{3}\right), \alpha_{i} \in \mathbb{N}_{0}, \partial_{k}^{\alpha} f(k):=\partial_{k_{1}}^{\alpha_{1}} \partial_{k_{2}}^{\alpha_{2}} \partial_{k_{3}}^{\alpha_{3}} f(k)$ and $|\alpha|:=\alpha_{1}+\alpha_{2}+\alpha_{3}$.

18 Povzner, A.J.: The expansion of arbitrary functions in terms of eigenfunctions of the operator $-\Delta u=c u$, Math. Sbornik 32, 109-156 (1953); A.M.S. Translations, Series 2 60, 1-49 (1967).

${ }^{19}$ See e.g. Yoshida, K.: Functional Analysis, Springer-Verlag, New York, 1974.

${ }^{20}$ See e.g. Theorem XI.41.(d) in Reed, M. and Simon, B.: Methods of Modern Mathematical Physics III, Academic Press, London, 1979.

${ }^{21}$ Zemach, C. and Klein, A.: The Born Expansion in Non-Relativistic Quantum Theory, Il Nuovo Cimento X 6, 1078-1087 (1958).

22 Isozaki, H.: Differentiability of generalized Fourier transforms associated with Schrödinger operators, J. Math. Kyoto Univ. 25 789-806 (1985).

${ }^{23}$ See e.g. Theorems XI.47 and XI.48 in reference 20. 